\title{
A new iterative approximation scheme for Reich-Suzuki-type nonexpansive operators with an application
}

\author{
Austine Efut Ofem ${ }^{1}$, Hüseyin Işı $\mathrm{k}^{2^{*}} \mathbb{D}$, Faeem Ali ${ }^{3}$ and Junaid Ahmad $^{4}$
}

\section{"Correspondence:}

isikhuseyin76@gmail.com

${ }^{2}$ Department of Engineering

Science, Bandırma Onyedi Eylül

University, Bandırma 10200,

Balıkesir, Turkey

Full list of author information is

available at the end of the article

\section{Springer}

\begin{abstract}
In this article, we propose a faster iterative scheme, called the AH iterative scheme, for approximating fixed points of contractive-like mappings and Reich-Suzuki-type nonexpansive mappings. We show that the AH iterative scheme converges faster than a number of existing iterative schemes for contractive-like mappings. The $w^{2}$-stability result of the new iterative scheme is established and a supportive example is provided to illustrate the notion of $w^{2}$-stability. Then, we prove weak and several strong convergence results of our new iterative scheme for fixed points of Reich-Suzuki-type nonexpansive mappings. Further, we carry out a numerical experiment to illustrate the efficiency of our new iterative scheme. As an application, we use our main result to prove the existence of a solution of a mixed-type nonlinear integral equation. An illustrative example to validate the result in our application is also given. Our results extend and generalize several related results in the existing literature.
\end{abstract}

MSC: $47 \mathrm{H} 05 ; 47 \mathrm{H09} ; 39 \mathrm{~B} 82$

Keywords: Banach space; $w^{2}$-stability; Contractive-like mapping; Iterative scheme; Nonlinear integral equation

\section{Introduction}

Throughout the paper, let $\mathbb{N}$ be the set of all natural numbers, $\mathbb{R}$ a set of all real numbers, $\mathcal{V}$ a nonempty subset of a Banach space $\mathcal{M}$. A mapping $U: \mathcal{V} \rightarrow \mathcal{V}$ is called a contraction if there exists $\zeta \in[0,1)$ such that $\|U p-U q\| \leq \zeta\|p-q\|$, for all $p, q \in \mathcal{V}$. If $\zeta=1$, then $U$ is called a nonexpansive mapping. A point $p^{\star} \in \mathcal{V}$ is said to be a fixed point of $U$ if it satisfies $U p^{\star}=p^{\star}$. We denote by $\Im(\mathcal{U})$ the set of all fixed points of $\mathcal{U}$.

The major ideas of fixed-point theory can be divided into two categories. One is to find the necessary and sufficient conditions under which an operator admits fixed points. The other is to determine such fixed points by using some schematic algorithms. Formally, the first category is usually referred to as the existence part and the second one is known as the computation or approximation part. Another important concept of fixed-point theory that is less well known is the study of the behaviors of fixed points such as stability, data dependency, etc.

(c) The Author(s) 2022. This article is licensed under a Creative Commons Attribution 4.0 International License, which permits use sharing, adaptation, distribution and reproduction in any medium or format, as long as you give appropriate credit to the original author(s) and the source, provide a link to the Creative Commons licence, and indicate if changes were made. The images or other third party material in this article are included in the article's Creative Commons licence, unless indicated otherwise in a credit line to the material. If material is not included in the article's Creative Commons licence and your intended use is not permitted by statutory regulation or exceeds the permitted use, you will need to obtain permission directly from the copyright holder. To view a copy of this licence, visit http://creativecommons.org/licenses/by/4.0/. 
For some decades now, the fixed-point theory has been revealed as a very powerful and useful tool in the study of nonlinear phenomenon. In particular, fixed-point techniques have been applied in diverse areas of biology, chemistry, economics, engineering, game theory, physics, etc., (see $[4,5,13,23-26,30,31]$ and the references therein).

In [6], Berinde provided the class of weak contractions that properly includes the class of Zamfirescu operators [47]. This class of mappings is also known by many authors as almost contraction mappings.

Definition 1.1 A mapping $U: \mathcal{V} \rightarrow \mathcal{V}$ is called a weak contraction if there exist $\zeta \in(0,1)$ and $L \geq 0$ such that

$$
\|u p-U q\| \leq \zeta\|p-q\|+L\|p-U p\|, \quad \text { for all } p, q \in \mathcal{V} .
$$

In [17], Imoru and Olantiwo gave a definition of a class of mapping considered to be a generalization of the classes of mappings studied by Berinde [6], Osilike and Udomene [28] and some other existing classes of contraction mappings as follows.

Definition $1.2([17])$ A mapping $U: \mathcal{V} \rightarrow \mathcal{V}$ is called contractive-like if there exists a constant $\zeta \in[0,1)$ and a strictly increasing continuous function $\psi:[0, \infty) \rightarrow[0, \infty)$ with $\psi(0)=0$ such that

$$
\left\|u_{p}-u_{q}\right\| \leq \zeta\|p-q\|+\psi(\|p-u p\|)
$$

for all $p, q \in \mathcal{V}$.

Remark 1.3 If $\psi(p)=L p$, then (1.2) reduces to (1.1).

In recent years, many extensions and generalizations of nonexpansive mappings have been studied by several authors due to their importance in terms of applications.

In 2008, Suzuki [35] introduced an interesting generalization of nonexpansive mappings and obtained some existence and convergence results. Such mappings are commonly known as mappings satisfying condition (C).

Definition 1.4 A mapping $U: \mathcal{V} \rightarrow \mathcal{V}$ is said to satisfy condition (C) if

$$
\frac{1}{2}\|p-U p\| \leq\|p-q\| \quad \text { implies } \quad\|u p-U q\| \leq\|p-q\|, \quad \text { for all } p, q \in \mathcal{V} .
$$

In 2019, Pant and Pandey [29] considered the class of Reich-Suzuki-type nonexpansive mappings as follows.

Definition 1.5 A mapping $U: \mathcal{V} \rightarrow \mathcal{V}$ is said to be Reich-Suzuki-type nonexpansive if there exists a real number $\varpi \in[0,1)$ such that for each $p, q \in \mathcal{V}$,

$$
\begin{aligned}
& \frac{1}{2}\left\|p-u_{p}\right\| \leq\|p-q\| \quad \text { implies } \\
& \left\|u_{p}-u_{q}\right\| \leq \varpi\|p-u p\|+\varpi\|q-U q\|+(1-2 \varpi)\|p-q\| .
\end{aligned}
$$


Clearly, every mapping satisfying condition (C) is a Reich-Suzuki-type nonexpansive mapping with $\varpi=0$. However, the converse is not true, as shown in [29].

On the other hand, many iterative schemes have been constructed to approximate the fixed points of different classes of mappings. Some of these iterative schemes are: Mann [21], Ishikawa [19], Noor [22], Agarwal et al. [2], Abbas and Nazir [1], CR [11], Normal-S [32], Picard-S [13], Thakur et al. [37], and M [41] iterative schemes.

Very recently, Ahmad et al. [3] introduced a novel iterative scheme known as the JK iterative scheme as follows:

$$
\left\{\begin{array}{l}
m_{1} \in \mathcal{V}, \\
\vartheta_{v}=\left(1-r_{v}\right) m_{v}+r_{v} U m_{v}, \\
\xi_{v}=U \vartheta_{v}, \\
m_{v+1}=U\left(\left(1-k_{v}\right) U \vartheta_{v}+k_{v} U \xi_{v}\right),
\end{array} \quad v \in \mathbb{N},\right.
$$

where $\left\{r_{v}\right\}$ and $\left\{k_{v}\right\}$ are sequences in $(0,1)$. The authors established some weak and strong convergence results for mappings satisfying condition $(C)$. They further showed numerically that the JK iterative scheme converges faster than the S [2] and Thakur [37] iterative schemes.

To see recent results concerning iterative schemes and the existence theory of fixed points one can refer to, for example, [10, 36, 43-46].

Motivated and inspired by the research in this direction, we propose a new fourstep iterative scheme called the AH iterative scheme, to approximate the fixed points of contractive-like mappings and Reich-Suzuki-type nonexpansive mappings as follows:

$$
\left\{\begin{array}{l}
p_{1} \in \mathcal{V}, \\
z_{v}=\left(1-r_{v}\right) p_{v}+r_{v} U p_{v}, \\
w_{v}=U\left(U z_{v}\right), \\
q_{v}=U\left(U w_{v}\right), \\
p_{v+1}=\left(1-k_{v}\right) q_{v}+k_{\nu} U q_{v},
\end{array} \quad v \in \mathbb{N},\right.
$$

where $\left\{r_{v}\right\}$ and $\left\{k_{v}\right\}$ are sequences in $(0,1)$.

The purpose of this article is to prove that the AH iterative scheme (1.6) converges faster than the JK iterative scheme (1.5) for contractive-like mappings. Numerically, we further show that the AH iterative scheme (1.6) converges faster than a number of existing iterative schemes. Also, we prove that our proposed iterative scheme defined by (1.6) is $w^{2}$-stable and the stability result is supported with an example. Again, we establish weak and strong convergence results of the AH iterative scheme (1.6) for Reich-Suzuki-type nonexpansive mappings. Further, we use a new example of Reich-Suzuki-type nonexpansive mappings to show that the $\mathrm{AH}$ iterative scheme (1.6) outperforms some existing prominent iterative schemes. Finally, we use our main results to establish the existence of the solution of a nonlinear integral equation in Banach spaces.

\section{Preliminaries}

Let $\mathcal{M}^{*}$ be the dual of a Banach space $\mathcal{M}$ and $\langle\cdot, \cdot\rangle$ denotes the generalized duality pairing between $\mathcal{M}$ and $\mathcal{M}^{*}$. Then, the multivalued mapping $J: \mathcal{M} \rightarrow 2^{\mathcal{M}^{*}}$ is the normalized 
duality mapping defined for each $p \in \mathcal{M}$ by

$$
J(p)=\left\{q \in \mathcal{M}^{*}:\langle p, q\rangle=\|p\|^{2}=\|q\|^{2}\right\} .
$$

Let $D=\{p \in \mathcal{M}:\|p\|=1\}$. Then, a Banach space $\mathcal{M}$ is said to be smooth if the limit

$$
\lim _{c \rightarrow 0} \frac{\|p+c q\|-\|p\|}{c}
$$

exists for each $p, q \in D$. In this case, the norm of $\mathcal{M}$ is called Gâteaux differentiable. It is well known that $J$ is single valued if $\mathcal{M}$ is smooth [12]. Suppose for each $p \in D$, the limit of (2.2) exists and is attained uniformly for $q \in D$, the norm of $\mathcal{M}$ is called Fréchet differentiable in this case. It is also well known that

$$
\langle q, J(p)\rangle+\frac{1}{2}\|p\|^{2} \leq \frac{1}{2}\|p+q\|^{2} \leq\langle q, J(p)\rangle+\frac{1}{2}\|p\|^{2}+b(q)
$$

for all $p, q \in \mathcal{M}$, where $J$ is the Fréchet derivative of the functional $\frac{1}{2}\|\cdot\|^{2}$ at $p \in \mathcal{M}$ and $b$ is an increasing function defined on $[0, \infty)$ such that $\lim _{v \downarrow 0} \frac{b(v)}{v}=0$.

A Banach space $\mathcal{M}$ is said to be uniformly convex if for each $\epsilon \in(0,2]$, there exists $\delta>0$ such that for $p, q \in \mathcal{M}$ satisfying $\|p\| \leq 1,\|q\| \leq 1$ and $\|p-q\|>\epsilon$, we have $\left\|\frac{p+q}{2}\right\|<1-\delta$.

A Banach space $\mathcal{M}$ is said to satisfy Opial's condition if for any sequence $\left\{p_{\nu}\right\}$ in $\mathcal{M}$ that converges weakly to $p \in \mathcal{M}$ implies

$$
\limsup _{v \rightarrow \infty}\left\|p_{v}-p\right\|<\limsup _{v \rightarrow \infty}\left\|p_{v}-q\right\|, \quad \forall q \in \mathcal{M} \text { with } q \neq p .
$$

Let $\mathcal{V}$ be a nonempty closed convex subset of a Banach space $\mathcal{M}$, and $\left\{p_{v}\right\}$ is a bounded sequence in $\mathcal{M}$. For $p \in \mathcal{M}$, we put

$$
r\left(p,\left\{p_{v}\right\}\right)=\limsup _{v \rightarrow \infty}\left\|p_{v}-p\right\| .
$$

The asymptotic radius of $\left\{p_{\nu}\right\}$ relative to $\mathcal{V}$ is defined by

$$
r\left(\mathcal{V},\left\{p_{v}\right\}\right)=\inf \left\{r\left(p,\left\{p_{\nu}\right\}\right): p \in \mathcal{V}\right\} .
$$

The asymptotic center of $\left\{p_{\nu}\right\}$ relative to $\mathcal{V}$ is given as:

$$
A\left(\mathcal{V},\left\{p_{v}\right\}\right)=\left\{p \in \mathcal{V}: r\left(p,\left\{p_{v}\right\}\right)=r\left(\mathcal{V},\left\{p_{v}\right\}\right)\right\}
$$

In a uniformly convex Banach space, it is well known that $A\left(\mathcal{V},\left\{p_{v}\right\}\right)$ consists of exactly one point.

Let $\mathcal{V}$ be a nonempty closed convex subset of a Banach space $\mathcal{M}$. A mapping $\mathcal{U}: \mathcal{V} \rightarrow \mathcal{V}$ is said to be demiclosed with respect to $p \in \mathcal{M}$, if for each sequence $\left\{p_{v}\right\}$ that is weakly convergent to $p \in \mathcal{V}$ and $\left\{u_{p}\right\}$ converges strongly to $q$ implies that $U_{p}=q$.

Definition 2.1 ([7]) Let $\left\{\delta_{v}\right\}$ and $\left\{\gamma_{v}\right\}$ be two sequences of real numbers that converge to $\delta$ and $\gamma$, respectively, and assume that there exists

$$
\ell=\lim _{\nu \rightarrow \infty} \frac{\left\|\delta_{v}-\delta\right\|}{\left\|\gamma_{v}-\gamma\right\|}
$$


Then,

$\left(\Theta_{1}\right)$ if $\ell=0$, we say that $\left\{\delta_{v}\right\}$ converges to $\delta$ faster than $\left\{\gamma_{v}\right\}$ does to $\gamma$.

$\left(\Theta_{2}\right)$ If $0<\ell<\infty$, we say that $\left\{\delta_{\nu}\right\}$ and $\left\{\gamma_{\nu}\right\}$ have the same rate of convergence.

Definition 2.2 ([7]) Let $\left\{\eta_{v}\right\}$ and $\left\{\phi_{v}\right\}$ be two fixed-point iteration processes that converge to the same point $p^{\star}$, the error estimates

$$
\begin{aligned}
& \left\|\eta_{v}-p^{\star}\right\| \leq \delta_{v}, \quad v \in \mathbb{N}, \\
& \left\|\phi_{v}-p^{\star}\right\| \leq \gamma_{v}, \quad v \in \mathbb{N}
\end{aligned}
$$

are available, where $\left\{\delta_{v}\right\}$ and $\left\{\gamma_{\nu}\right\}$ are two sequences of positive numbers converging to zero. Then, we say that $\left\{\eta_{\nu}\right\}$ converges faster to $p^{\star}$ than $\left\{\phi_{\nu}\right\}$ does if $\left\{\delta_{v}\right\}$ converges faster than $\left\{\gamma_{v}\right\}$.

Definition 2.3 A sequence $\left\{p_{v}\right\}$ in $\mathcal{V}$ is said to be an approximate fixed-point sequence (a.f.p.s. for short) for a mapping $\mathcal{U}: \mathcal{V} \rightarrow \mathcal{V}$ if

$$
\lim _{v \rightarrow \infty}\left\|U p_{v}-p_{v}\right\|=0
$$

Definition 2.4 ([34]) A mapping $U: \mathcal{V} \rightarrow \mathcal{V}$ is said to be a satisfied condition $(I)$ if a nondecreasing function $f:[0, \infty) \rightarrow[0, \infty)$ exists with $f(0)=0$ and for all $s>0$, then $f(s)>$ 0 such that $\|p-\mathcal{U} p\| \geq f(d(p, \Im(U)))$ for all $p \in \mathcal{V}$, where $d(p, \Im(U))=\inf _{p^{\star} \in \Im(u)}\left\|p-p^{\star}\right\|$.

Lemma 2.5 ([42]) Let $\left\{\theta_{v}\right\}$ and $\left\{\lambda_{v}\right\}$ be nonnegative real sequences satisfying the following inequalities:

$$
\theta_{v+1} \leq\left(1-\sigma_{v}\right) \theta_{v}+\lambda_{v}
$$

where $\sigma_{v} \in(0,1)$ for all $v \in \mathbb{N}, \sum_{v=0}^{\infty} \sigma_{v}=\infty$ and $\lim _{v \rightarrow \infty} \frac{\lambda_{v}}{\sigma_{v}}=0$, then $\lim _{v \rightarrow \infty} \theta_{v}=0$.

Lemma 2.6 ([33]) Suppose $\mathcal{M}$ is a uniformly convex Banach space and $\left\{\iota_{v}\right\}$ is any sequence satisfying $0<p \leq \iota_{v} \leq q<1$ for all $v \geq 1$. Suppose $\left\{p_{v}\right\}$ and $\left\{q_{v}\right\}$ are any sequences of $\mathcal{M}$ such that

$$
\begin{aligned}
& \limsup _{v \rightarrow \infty}\left\|p_{v}\right\| \leq x, \\
& \limsup _{v \rightarrow \infty}\left\|q_{v}\right\| \leq x \quad \text { and } \\
& \limsup _{v \rightarrow \infty}\left\|\iota_{v} p_{v}+\left(1-\iota_{v}\right) q_{v}\right\|=x
\end{aligned}
$$

hold for some $x \geq 0$. Then, $\lim _{v \rightarrow \infty}\left\|p_{v}-q_{v}\right\|=0$.

Lemma 2.7 ([39]) Let $\mathcal{U}: \mathcal{V} \rightarrow \mathcal{V}$ be a mapping. If $U$ is a Reich-Suzuki-type nonexpansive mapping with $\Im(\mathcal{\Im}) \neq \emptyset$, then the following hold:

(i) If $\mathcal{U}$ is a Reich-Suzuki-type nonexpansive mapping, then for every choice of $p \in \mathcal{V}$ and $p^{\star} \in \Im(U)$, it follows that $\left\|u p-U p^{\star}\right\| \leq\left\|p-p^{\star}\right\|$.

(ii) If $\mathcal{U}$ satisfies condition $(C)$, then $\mathcal{U}$ is a Reich-Suzuki-type nonexpansive mapping. 
Lemma 2.8 ([40]) Let $\mathcal{U}: \mathcal{V} \rightarrow \mathcal{V}$ be a mapping. If $\mathcal{U}$ is a Reich-Suzuki-type nonexpansive mapping, then for all $p, q \in \mathcal{V}$, the following inequality holds:

$$
\|p-u q\| \leq\left(\frac{3+\varpi}{1-\varpi}\right)\|p-u p\|+\|p-q\| .
$$

We now offer a numerical example that satisfies the inequality of the above lemma but does not satisfy the condition $(\mathrm{C})$.

Example 2.9 Let $(\mathbb{R},\|\cdot\|)$ be a Banach space with the usual norm and $\mathcal{V}=[-1,1]$. Define $\mathcal{U}: \mathcal{V} \rightarrow \mathcal{V}$ by

$$
U_{p}= \begin{cases}\frac{-p}{3}, & \text { if } p \in[-1,0), \\ -p, & \text { if } p \in[0,1] \backslash\left\{\frac{1}{3}\right\}, \\ 0, & \text { if } p \in\left\{\frac{1}{3}\right\} .\end{cases}
$$

(1) The mapping $U$ does not satisfy the condition (C) and hence is not a nonexpansive mapping. If we take $p=\frac{1}{3}$ and $q=1$, then

$$
\frac{1}{2}|p-u p|=\frac{1}{2}\left|\frac{1}{3}-u\left(\frac{1}{3}\right)\right|=\frac{1}{6} \leq \frac{2}{3}=|p-q| .
$$

On the other hand,

$$
\left|u_{p}-u_{q}\right|=1>\frac{2}{3}=|p-q| .
$$

(2) Now, we show that $U$ satisfies condition (2.4). For this, the following conditions are considered:

Case I: If $p, q \in[-1,0)$, we have

$$
\begin{aligned}
\left|p-u_{q}\right| & \leq\left|p-u_{p}\right|+\left|u_{p}-u_{q}\right| \\
& =\left|p-u_{p}\right|+\frac{1}{3}|p-q| .
\end{aligned}
$$

Case II: If $p, q \in[0,1] \backslash\left\{\frac{1}{3}\right\}$, then we obtain

$$
\begin{aligned}
|p-U q| & \leq|p-u p|+|u p-U q| \\
& \leq\left|p-u_{p}\right|+|p-q| .
\end{aligned}
$$

Case III: If $p \in[-1,0)$ and $q \in[0,1] \backslash\left\{\frac{1}{3}\right\}$, we obtain

$$
\begin{aligned}
|p-U q| & =|p+q| \leq|p|+|q| \\
& \leq \frac{4}{3}|p|+|p-q| \quad(\text { as } p<0 \text { and } q \geq 0) \\
& =\left|p-\left(-\frac{p}{3}\right)\right|+|p-q| \\
& =|p-U q|+|p-q| .
\end{aligned}
$$


Case IV: If $p \in[-1,0)$ and $q=\frac{1}{3}$, we have

$$
\begin{aligned}
|p-U q| & =|p| \leq \frac{4}{3}|p|+\left|p-\frac{1}{3}\right| \\
& =|p-U p|+|p-q| .
\end{aligned}
$$

Case V: If $p \in[0,1] \backslash\left\{\frac{1}{3}\right\}$ and $q=\frac{1}{3}$, we obtain

$$
\begin{aligned}
|p-U q| & =|p| \leq 2|p|+\left|p-\frac{1}{3}\right| \\
& =|p-U q|+\left|p-\frac{1}{3}\right|
\end{aligned}
$$

Hence, $\mathcal{U}$ satisfies the condition (2.4) with $\frac{3+\varpi}{1-\varpi} \geq 1$.

\section{Rate of convergence}

In this section, we show both analytically and numerically that the AH iterative scheme (1.6) converges faster than the JK iterative scheme (1.5) for contractive-like mappings.

Theorem 3.1 Let $U$ be a mapping satisfying (1.2) defined on a nonempty closed convex subset $\mathcal{V}$ of a Banach space $\mathcal{M}$. Then, the sequence $\left\{p_{v}\right\}$ generated by the AH iterative scheme (1.6) converges strongly to a unique fixed point of $u$.

Proof Using (1.2) and (1.6), we have

$$
\begin{aligned}
\left\|z_{v}-p^{\star}\right\| & =\left\|\left(1-r_{v}\right) p_{v}+r_{v} u p_{v}-u p^{\star}\right\| \\
& \leq\left(1-r_{v}\right)\left\|p_{v}-p^{\star}\right\|+r_{v}\left\|u p_{v}-u p^{\star}\right\| \\
& \leq\left(1-r_{v}\right)\left\|p_{v}-p^{\star}\right\|+r_{v}\left[\zeta\left\|p_{v}-p^{\star}\right\|+L\left\|p^{\star}-u p^{\star}\right\|\right] \\
& =\left(1-r_{v}(1-\zeta)\right)\left\|p_{v}-p^{\star}\right\| .
\end{aligned}
$$

Using (1.6) and (3.1), we have

$$
\begin{aligned}
\left\|w_{v}-p^{\star}\right\| & =\left\|u\left(U z_{v}\right)-p^{\star}\right\| \\
& \leq \zeta\left\|U z_{v}-p^{\star}\right\| \\
& \leq \zeta^{2}\left\|z_{v}-p^{\star}\right\| \\
& \leq \zeta^{2}\left(1-r_{v}(1-\zeta)\right)\left\|p_{v}-p^{\star}\right\| .
\end{aligned}
$$

Now, from (1.6) and (3.2), we obtain

$$
\begin{aligned}
\left\|q_{v}-p^{\star}\right\| & =\left\|U\left(U_{w_{v}}\right)-p^{\star}\right\| \\
& \leq \zeta\left\|U w_{v}-p^{\star}\right\| \\
& \leq \zeta^{2}\left\|w_{v}-p^{\star}\right\| \\
& \leq \zeta^{4}\left(1-r_{v}(1-\zeta)\right)\left\|p_{v}-p^{\star}\right\| .
\end{aligned}
$$


Finally, using (1.6) and (3.3), we obtain

$$
\begin{aligned}
\left\|p_{v+1}-p^{\star}\right\| & =\left\|\left(1-k_{v}\right) q_{v}+k_{v} U q_{v}-U p^{\star}\right\| \\
& \leq\left(1-k_{v}\right)\left\|q_{v}-p^{\star}\right\|+k_{v}\left\|u q_{v}-U p^{\star}\right\| \\
& \leq\left(1-k_{v}(1-\zeta)\right)\left\|q_{v}-p^{\star}\right\| \\
& \leq \zeta^{4}\left(1-r_{v}(1-\zeta)\right)\left(1-k_{v}(1-\zeta)\right)\left\|p_{v}-p^{\star}\right\| .
\end{aligned}
$$

Since $0<\zeta<1$ and $r_{v}, k_{v} \in(0,1)$, it implies that $\left(1-r_{v}(1-\zeta)\right)<1$ and $\left(1-k_{\nu}(1-\zeta)\right)<1$. It follows that $\left(1-k_{v}(1-\zeta)\right)\left(1-k_{v}(1-\zeta)\right)<1$.

Thus, from (3.4), we have

$$
\left\|p_{v+1}-p^{\star}\right\| \leq \zeta^{4}\left\|p_{v}-p^{\star}\right\|
$$

Inductively, we obtain:

$$
\left\|p_{v+1}-p^{\star}\right\| \leq \zeta^{4(v+1)}\left\|p_{0}-p^{\star}\right\|
$$

Since $0<\zeta<1, p_{v} \rightarrow p^{\star}$ as $v \rightarrow \infty$. This completes the proof.

Theorem 3.2 Let $U$ be a mapping satisfying (1.2) defined on a nonempty closed convex subset $\mathcal{V}$ of a Banach space $\mathcal{M}$. If $\left\{p_{v}\right\}$ is a sequence generated by the AH iterative scheme (1.6), then $\left\{p_{v}\right\}$ converges faster than $\left\{m_{v}\right\}$ generated by the JK iterative scheme (1.5).

Proof Recalling (3.6) in Theorem 3.1, we have

$$
\left\|p_{v+1}-p^{\star}\right\| \leq \zeta^{4(v+1)}\left\|p_{0}-p^{\star}\right\|, \quad v \in \mathbb{N}
$$

Also, from (1.5), we obtain

$$
\begin{aligned}
\left\|\vartheta_{v}-p^{\star}\right\| & =\left\|\left(1-r_{v}\right) m_{v}+r_{v} U m_{v}-U p^{\star}\right\| \\
& \leq\left(1-r_{v}\right)\left\|m_{v}-p^{\star}\right\|+r_{v}\left\|U m_{v}-U p^{\star}\right\| \\
& =\left(1-r_{v}(1-\zeta)\right)\left\|m_{v}-p^{\star}\right\| .
\end{aligned}
$$

Using (1.5) and (3.7), we have

$$
\begin{aligned}
\left\|\xi_{v}-p^{\star}\right\| & =\left\|U \vartheta_{v}-p^{\star}\right\| \\
& \leq \zeta\left\|\vartheta_{v}-p^{\star}\right\| \\
& \leq \zeta\left(1-r_{v}(1-\zeta)\right)\left\|m_{v}-p^{\star}\right\| .
\end{aligned}
$$

Again, from (1.5) and (3.8), we obtain

$$
\left\|m_{v+1}-p^{\star}\right\|=\left\|u\left(\left(1-k_{v}\right) u \vartheta_{v}+k_{v} u \xi_{v}\right)-u p^{\star}\right\|
$$




$$
\begin{aligned}
\leq & \zeta\left(\left(1-k_{v}\right)\left\|U \vartheta_{v}-U p^{\star}\right\|+k_{v}\left\|u \xi_{v}-U p^{\star}\right\|\right) \\
\leq & \zeta^{2}\left(\left(1-k_{v}\right)\left\|\vartheta_{v}-p^{\star}\right\|+k_{v}\left\|\xi_{v}-p^{\star}\right\|\right) \\
\leq & \zeta^{2}\left(\left(1-k_{v}\right)\left(1-r_{v}(1-\zeta)\right)\left\|m_{v}-p^{\star}\right\|\right. \\
& \left.+k_{\nu} \zeta\left(1-r_{v}(1-\zeta)\right)\left\|m_{v}-p^{\star}\right\|\right) \\
= & \zeta^{2}\left(\left(1-r_{v}(1-\zeta)\right)\left(1-k_{v}(1-\zeta)\right)\right)\left\|m_{v}-p^{\star}\right\| \\
\leq & \zeta^{2}\left\|m_{v}-p^{\star}\right\| .
\end{aligned}
$$

Inductively, we obtain:

$$
\left\|m_{v+1}-p^{\star}\right\| \leq \zeta^{2(v+1)}\left\|m_{0}-p^{\star}\right\| .
$$

Let $\Lambda_{v}=\zeta^{4(v+1)}\left\|p_{0}-p^{\star}\right\|$ and $\beta_{v}=\zeta^{2(v+1)}\left\|m_{0}-p^{\star}\right\|$, then we have that

$$
\frac{\Lambda_{v}}{\beta_{v}}=\frac{\zeta^{4(v+1)}\left\|p_{0}-p^{\star}\right\|}{\zeta^{2(v+1)}\left\|m_{0}-p^{\star}\right\|}=\zeta^{2(v+1)} \frac{\left\|p_{0}-p^{\star}\right\|}{\left\|m_{0}-p^{\star}\right\|} \rightarrow 0 \quad \text { as } v \rightarrow \infty .
$$

Hence, the sequence $\left\{p_{v}\right\}$ converges faster to $p^{\star}$ than $\left\{m_{v}\right\}$.

Now, we give a nontrivial example to compare the rate of convergence of the AH iterative scheme (1.6) with some leading iterative schemes in the literature.

Example 3.3 Let $\mathcal{M}=\mathbb{R}^{3}$ and $\mathcal{V}=\left\{p=\left(p_{1}, p_{2}, p_{3}\right):\left(p_{1}, p_{2}, p_{3}\right) \in[0,8] \times[0,8] \times[0,8]\right\}$ be a subset of $\mathcal{M}$ with norm $\|p\|=\left\|\left(p_{1}, p_{2}, p_{3}\right)\right\|=\left|p_{1}\right|+\left|p_{2}\right|+\left|p_{3}\right|$. Let $\mathcal{U}: \mathcal{V} \rightarrow \mathcal{V}$ be defined by

$$
U\left(p_{1}, p_{2}, p_{3}\right)= \begin{cases}\left(\frac{p_{1}}{4}, \frac{p_{2}}{4}, \frac{p_{3}}{4}\right), & \text { if }\left(p_{1}, p_{2}, p_{3}\right) \in[0,4) \times[0,4) \times[0,4), \\ \left(\frac{p_{1}}{8}, \frac{p_{2}}{8}, \frac{p_{3}}{8}\right), & \text { if }\left(p_{1}, p_{2}, p_{3}\right) \in[4,8] \times[4,8] \times[4,8] .\end{cases}
$$

Clearly, the only fixed point of $U$ is $(0,0,0)$. We will now show that $U$ is a contractive-like mapping. To see this, we define a function $\psi:[0, \infty) \rightarrow[0, \infty)$ by $\psi(p)=\frac{p}{6}$. Clearly, $\psi$ is a strictly increasing continuous function with $\psi(0)=0$. We show that

$$
\left\|u_{p}-u_{p}\right\|=\zeta\|p-q\|+\psi\left(\left\|p-u_{p}\right\|\right)
$$

for all $p, q \in \mathcal{V}$ and $\zeta \in(0,1]$. It will be useful to note the following. If $p=\left(p_{1}, p_{2}, p_{3}\right) \in$ $[0,4) \times[0,4) \times[0,4)$, then

$$
\|p-u p\|=\left\|\left(p_{1}, p_{2}, p_{3}\right)-\left(\frac{p_{1}}{4}, \frac{p_{2}}{4}, \frac{p_{3}}{4}\right)\right\|=\left\|\left(\frac{3 p_{1}}{4}, \frac{3 p_{2}}{4}, \frac{3 p_{3}}{4}\right)\right\|
$$

and

$$
\begin{aligned}
\psi(\|p-u p\|) & =\psi\left(\left\|\left(\frac{3 p_{1}}{4}, \frac{3 p_{2}}{4}, \frac{3 p_{3}}{4}\right)\right\|\right) \\
& =\left\|\left(\frac{p_{1}}{8}, \frac{p_{2}}{8}, \frac{p_{3}}{8}\right)\right\|=\left|\frac{p_{1}}{8}\right|+\left|\frac{p_{2}}{8}\right|+\left|\frac{p_{3}}{8}\right| .
\end{aligned}
$$


Similarly, if $p=\left(p_{1}, p_{2}, p_{3}\right) \in[4,8] \times[4,8] \times[4,8]$, we have

$$
\|p-u p\|=\left\|\left(p_{1}, p_{2}, p_{3}\right)-\left(\frac{p_{1}}{8}, \frac{p_{2}}{8}, \frac{p_{3}}{8}\right)\right\|=\left\|\left(\frac{7 p_{1}}{8}, \frac{7 p_{2}}{8}, \frac{7 p_{3}}{8}\right)\right\|
$$

and

$$
\begin{aligned}
\psi\left(\left\|p-u_{p}\right\|\right) & =\psi\left(\left\|\left(\frac{7 p_{1}}{8}, \frac{7 p_{2}}{8}, \frac{7 p_{3}}{8}\right)\right\|\right) \\
& =\left\|\left(\frac{7 p_{1}}{48}, \frac{7 p_{2}}{48}, \frac{7 p_{3}}{48}\right)\right\|=\left|\frac{7 p_{1}}{48}\right|+\left|\frac{7 p_{2}}{48}\right|+\left|\frac{7 p_{3}}{48}\right| .
\end{aligned}
$$

Next, we consider the following cases:

Case 1: If $p=\left(p_{1}, p_{2}, p_{3}\right), q=\left(q_{1}, q_{2}, q_{3}\right) \in[0,4) \times[0,4) \times[0,4)$, then using (3.13), we have

$$
\begin{aligned}
\left\|u_{p}-u_{q}\right\| & =\left\|\left(\frac{p_{1}}{4}, \frac{p_{2}}{4}, \frac{p_{3}}{4}\right)-\left(\frac{q_{1}}{4}, \frac{q_{2}}{4}, \frac{q_{3}}{4}\right)\right\| \\
& =\left|\frac{p_{1}}{4}-\frac{q_{1}}{4}\right|+\left|\frac{p_{2}}{4}-\frac{q_{2}}{4}\right|+\left|\frac{p_{3}}{4}-\frac{q_{3}}{4}\right| \\
& =\frac{1}{4}\left|p_{1}-q_{1}\right|+\frac{1}{4}\left|p_{2}-q_{2}\right|+\frac{1}{4}\left|p_{3}-q_{3}\right| \\
& =\frac{1}{4}\left\|\left(p_{1}, p_{2}, p_{3}\right)-\left(q_{1}, q_{2}, q_{3}\right)\right\| \\
& \leq \frac{1}{4}\|p-q\|+\left|\frac{p_{1}}{8}\right|+\left|\frac{p_{2}}{8}\right|+\left|\frac{p_{3}}{8}\right| \\
& =\frac{1}{4}\|p-q\|+\psi\left(\left\|p-u_{p}\right\|\right) .
\end{aligned}
$$

Case 2: If $p=\left(p_{1}, p_{2}, p_{3}\right), q=\left(q_{1}, q_{2}, q_{3}\right) \in[4,8] \times[4,8] \times[4,8]$, then using (3.14), we obtain

$$
\begin{aligned}
\left\|u_{p}-u_{q}\right\| & =\left\|\left(\frac{p_{1}}{8}, \frac{p_{2}}{8}, \frac{p_{3}}{8}\right)-\left(\frac{q_{1}}{8}, \frac{q_{2}}{8}, \frac{q_{3}}{8}\right)\right\| \\
& =\left|\frac{p_{1}}{8}-\frac{q_{1}}{8}\right|+\left|\frac{p_{2}}{8}-\frac{q_{2}}{8}\right|+\left|\frac{p_{3}}{8}-\frac{q_{3}}{8}\right| \\
& =\frac{1}{8}\left|p_{1}-q_{1}\right|+\frac{1}{8}\left|p_{2}-q_{2}\right|+\frac{1}{8}\left|p_{3}-q_{3}\right| \\
& =\frac{1}{8}\left\|\left(p_{1}, p_{2}, p_{3}\right)-\left(q_{1}, q_{2}, q_{3}\right)\right\| \\
& \leq \frac{1}{4}\|p-q\|+\left|\frac{7 p_{1}}{48}\right|+\left|\frac{7 p_{2}}{48}\right|+\left|\frac{7 p_{3}}{48}\right| \\
& =\frac{1}{4}\|p-q\|+\psi\left(\left\|p-u_{p}\right\|\right) .
\end{aligned}
$$

Case 3: If $p=\left(p_{1}, p_{2}, p_{3}\right) \in[0,4) \times[0,4) \times[0,4)$ and $q=\left(q_{1}, q_{2}, q_{3}\right) \in[4,8] \times[4,8] \times[4,8]$, then using (3.13), we have

$$
\|u p-U q\|=\left\|\left(\frac{p_{1}}{4}, \frac{p_{2}}{4}, \frac{p_{3}}{4}\right)-\left(\frac{p_{1}}{8}, \frac{p_{2}}{8}, \frac{p_{3}}{8}\right)\right\|
$$




$$
\begin{aligned}
& =\left\|\left(\frac{p_{1}}{4}-\frac{q_{1}}{8}\right),\left(\frac{p_{2}}{4}-\frac{q_{2}}{8}\right),\left(\frac{p_{3}}{4}-\frac{q_{3}}{8}\right)\right\| \\
& =\left\|\left(\frac{p_{1}}{8}+\frac{p_{1}}{8}-\frac{q_{1}}{8}\right),\left(\frac{p_{2}}{8}+\frac{p_{2}}{8}-\frac{q_{2}}{8}\right),\left(\frac{p_{3}}{8}+\frac{p_{3}}{8}-\frac{q_{3}}{8}\right)\right\| \\
& =\left|\frac{p_{1}}{8}+\frac{p_{1}}{8}-\frac{q_{1}}{8}\right|+\left|\frac{p_{2}}{8}+\frac{p_{2}}{8}-\frac{q_{2}}{8}\right|+\left|\frac{p_{3}}{8}+\frac{p_{3}}{8}-\frac{q_{3}}{8}\right| \\
& \leq\left|\frac{p_{1}}{8}\right|+\left|\frac{p_{2}}{8}\right|+\left|\frac{p_{3}}{8}\right|+\left|\frac{p_{1}}{8}-\frac{q_{1}}{8}\right|+\left|\frac{p_{2}}{8}-\frac{q_{2}}{8}\right|+\left|\frac{p_{3}}{8}-\frac{q_{3}}{8}\right| \\
& =\frac{1}{8}\left(\left|p_{1}-q_{1}\right|+\left|p_{2}-q_{2}\right|+\left|p_{3}-q_{3}\right|\right)+\psi(\|p-U p\|) \\
& \leq \frac{1}{4}\left\|\left(p_{1}, p_{2}, p_{3}\right)-\left(q_{1}, q_{2}, q_{3}\right)\right\|+\psi\left(\left\|p-U_{p}\right\|\right) \\
& =\frac{1}{4}\|p-q\|+\psi\left(\left\|p-U_{p}\right\|\right) .
\end{aligned}
$$

Case 4: If $p=\left(p_{1}, p_{2}, p_{3}\right) \in[4,8] \times[4,8] \times[4,8]$ and $q=\left(q_{1}, q_{2}, q_{3}\right) \in[0,4) \times[0,4) \times[0,4)$, then using (3.13), we obtain

$$
\begin{aligned}
\left\|u_{p}-u_{q}\right\| & =\left\|\left(\frac{p_{1}}{8}, \frac{p_{2}}{8}, \frac{p_{3}}{8}\right)-\left(\frac{p_{1}}{4}, \frac{p_{2}}{4}, \frac{p_{3}}{4}\right)\right\| \\
& =\left\|\left(\frac{p_{1}}{8}-\frac{q_{1}}{4}\right),\left(\frac{p_{2}}{8}-\frac{q_{2}}{4}\right),\left(\frac{p_{3}}{8}-\frac{q_{3}}{4}\right)\right\| \\
& =\left\|\left(\frac{p_{1}}{4}-\frac{p_{1}}{8}-\frac{q_{1}}{4}\right),\left(\frac{p_{2}}{4}-\frac{p_{2}}{8}-\frac{q_{2}}{4}\right),\left(\frac{p_{3}}{4}-\frac{p_{3}}{8}-\frac{q_{3}}{4}\right)\right\| \\
& =\left|\frac{p_{1}}{4}-\frac{p_{1}}{8}-\frac{q_{1}}{4}\right|+\left|\frac{p_{2}}{4}-\frac{p_{2}}{8}-\frac{q_{2}}{4}\right|+\left|\frac{p_{3}}{4}-\frac{p_{3}}{8}-\frac{q_{3}}{4}\right| \\
& \leq\left|\frac{p_{1}}{8}\right|+\left|\frac{p_{2}}{8}\right|+\left|\frac{p_{3}}{8}\right|+\left|\frac{p_{1}}{4}-\frac{q_{1}}{4}\right|+\left|\frac{p_{2}}{4}-\frac{q_{2}}{4}\right|+\left|\frac{p_{3}}{4}-\frac{q_{3}}{4}\right| \\
& =\frac{1}{4}\left(\left|p_{1}-q_{1}\right|+\left|p_{2}-q_{2}\right|+\left|p_{3}-q_{3}\right|\right)+\psi\left(\left\|p-U_{p}\right\|\right) \\
& =\frac{1}{4}\left\|\left(p_{1}, p_{2}, p_{3}\right)-\left(q_{1}, q_{2}, q_{3}\right)\right\|+\psi\left(\left\|p-U_{p}\right\|\right) \\
& =\frac{1}{4}\|p-q\|+\psi\left(\left\|p-U_{p}\right\|\right) .
\end{aligned}
$$

Hence, (5.1) is fulfilled with $\zeta=\frac{1}{4}$. Thus, $\mathcal{U}$ is a contractive-like mapping.

Using MATLAB R2015a, we obtain Tables 1-3 and Fig. 1. From Tables 1-3, we can easily see that all the iterative schemes with control parameters $r_{v}=0.8, k_{v}=0.6, l_{v}=0.5$, $v \in \mathbb{N}$ and starting point $(2,2.5,3)$ converge to $p^{\star}=(0,0,0)$. Obviously, our iterative scheme (1.6) requires the least number of iterations as compared to other iterative schemes. Also, from the graphical point of view in Fig. 1, it is evident that the AH iterative scheme (1.6) converges faster than other iterative schemes.

\section{Stability results}

The concept of stability of a fixed-point iteration process was rigorously studied by Harder in her Ph.D thesis that was published in 1987. 
Table 1 Convergence behavior of various iterative schemes

\begin{tabular}{cll}
\hline Step & Noor & $\mathrm{S}$ \\
\hline 1 & $(2.000000,2.500000,3.000000)$ & $(2.000000,2.500000,3.000000)$ \\
2 & $(0.597500,0.746875,0.896250)$ & $(0.320000,0.400000,0.480000)$ \\
3 & $(0.178503,0.223129,0.267755)$ & $(0.051200,0.064000,0.076800)$ \\
4 & $(0.053328,0.066660,0.079992)$ & $(0.008192,0.010240,0.012288)$ \\
5 & $(0.015932,0.019915,0.023898)$ & $(0.001311,0.001638,0.001966)$ \\
6 & $(0.004760,0.005949,0.007139)$ & $(0.000210,0.000262,0.000315)$ \\
7 & $(0.001422,0.001777,0.002133)$ & $(0.000034,0.000042,0.000050)$ \\
8 & $(0.000425,0.000531,0.000637)$ & $(0.000005,0.000007,0.000008)$ \\
9 & $(0.000127,0.000159,0.000190)$ & $(0.000001,0.000001,0.000001)$ \\
10 & $(0.000038,0.000047,0.000057)$ & $(0.000000,0.000000,0.000000)$ \\
11 & $(0.000011,0.000014,0.000017)$ & $(0.000000,0.000000,0.000000)$ \\
12 & $(0.000003,0.000004,0.000005)$ & $(0.000000,0.000000,0.000000)$ \\
13 & $(0.000001,0.000001,0.000002)$ & $(0.000000,0.000000,0.000000)$ \\
14 & $(0.000000,0.000000,0.000000)$ & $(0.000000,0.000000,0.000000)$
\end{tabular}

Table 2 Convergence behavior of various iterative schemes

\begin{tabular}{cll}
\hline Step & Abbas & Thakur \\
\hline 1 & $(2.000000,2.500000,3.000000)$ & $(2.000000,2.500000,3.000000)$ \\
2 & $(0.269375,0.336719,0.404062)$ & $(0.080000,0.100000,0.120000)$ \\
3 & $(0.036281,0.045352,0.054422)$ & $(0.003200,0.004000,0.004800)$ \\
4 & $(0.004887,0.006108,0.007330)$ & $(0.000128,0.000160,0.000192)$ \\
5 & $(0.000658,0.000823,0.000987)$ & $(0.000005,0.000006,0.000008)$ \\
6 & $(0.000089,0.000111,0.000133)$ & $(0.000000,0.000000,0.000000)$ \\
7 & $(0.000012,0.000015,0.000018)$ & $(0.000000,0.000000,0.000000)$ \\
8 & $(0.000002,0.000002,0.000002)$ & $(0.000000,0.000000,0.000000)$ \\
9 & $(0.000000,0.000000,0.000000)$ & $(0.000000,0.000000,0.000000)$ \\
10 & $(0.000000,0.000000,0.000000)$ & $(0.000000,0.000000,0.000000)$ \\
11 & $(0.000000,0.000000,0.000000)$ & $(0.000000,0.000000,0.000000)$ \\
12 & $(0.000000,0.000000,0.000000)$ & $(0.000000,0.000000,0.000000)$ \\
13 & $(0.000000,0.000000,0.000000)$ & $(0.000000,0.000000,0.000000)$ \\
14 & $(0.000000,0.000000,0.000000)$ & $(0.000000,0.000000,0.000000)$ \\
\hline
\end{tabular}

Table 3 Convergence behavior of various iterative schemes

\begin{tabular}{cll}
\hline Step & JK & AH \\
\hline 1 & $(2.000000,2.500000,3.000000)$ & $(2.000000,2.500000,3.000000)$ \\
2 & $(0.027500,0.034375,0.041250)$ & $(0.001719,0.002148,0.002578)$ \\
3 & $(0.000378,0.000473,0.000567)$ & $(0.000000,0.000000,0.000000)$ \\
4 & $(0.000005,0.000006,0.000008)$ & $(0.000000,0.000000,0.000000)$ \\
5 & $(0.000000,0.000000,0.000000)$ & $(0.000000,0.000000,0.000000)$ \\
6 & $(0.000000,0.000000,0.000000)$ & $(0.000000,0.000000,0.000000)$ \\
7 & $(0.000000,0.000000,0.000000)$ & $(0.000000,0.000000,0.000000)$ \\
8 & $(0.000000,0.000000,0.000000)$ & $(0.000000,0.000000,0.000000)$ \\
9 & $(0.000000,0.000000,0.000000)$ & $(0.000000,0.000000,0.000000)$ \\
10 & $(0.000000,0.000000,0.000000)$ & $(0.000000,0.000000,0.000000)$ \\
11 & $(0.000000,0.000000,0.000000)$ & $(0.000000,0.000000,0.000000)$ \\
12 & $(0.000000,0.000000,0.000000)$ & $(0.000000,0.000000,0.000000)$ \\
13 & $(0.000000,0.000000,0.000000)$ & $(0.000000,0.000000,0.000000)$ \\
14 & $(0.000000,0.000000,0.000000)$ & $(0.000000,0.000000,0.000000)$ \\
\hline
\end{tabular}

Definition 4.1 $([14,15])$ Let $\mathcal{U}: \mathcal{V} \rightarrow \mathcal{V}$ be a mapping. Define a fixed-point iteration method by $p_{v+1}=f\left(U, p_{v}\right)$ such that $\left\{p_{v}\right\}$ converges to a fixed point $p^{\star}$ of $U$. Let $\left\{h_{v}\right\}$ be an arbitrary sequence in $\mathcal{M}$. Define

$$
\epsilon_{v}=\left\|h_{v}-f\left(U, h_{v}\right)\right\|, \quad \forall v \in \mathbb{N}
$$




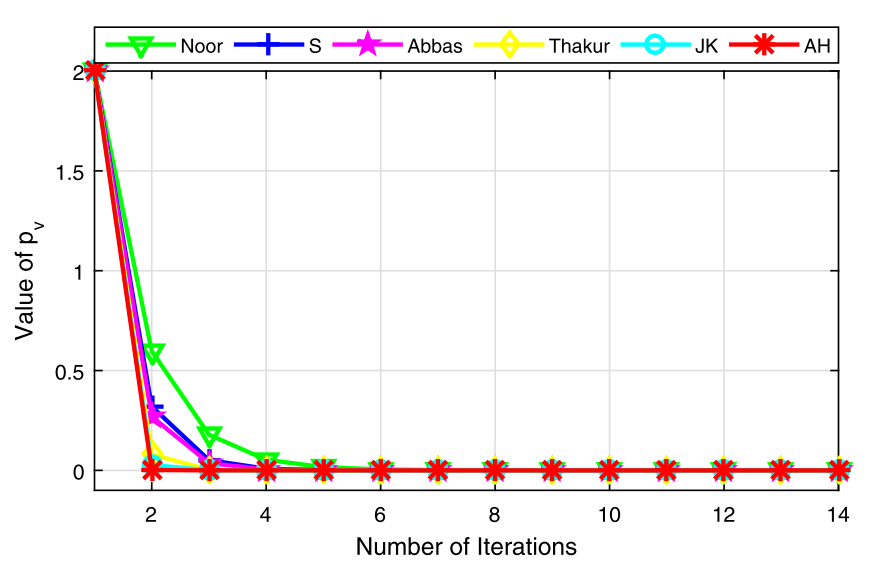

Figure 1 Graph corresponding to Tables 1-3

A fixed-point iterative method is said to be $U$-stable if the following condition is fulfilled:

$$
\lim _{v \rightarrow \infty} \epsilon_{\nu}=0 \quad \text { if and only if } \quad \lim _{\nu \rightarrow \infty} h_{\nu}=p^{\star} .
$$

The notion of stability in Definition 4.1 has recently been studied by several authors for different classes of contraction mappings (see $[16,18,27]$ and the references in them).

In [8], Berinde observed that the concept of stability in Definition 4.1 is not precise because of the sequence $\left\{h_{v}\right\}$ that is arbitrarily taken. To overcome this limitation, Berinde [8] observed that it would be more natural if $\left\{h_{\nu}\right\}$ were an approximate sequence of $\left\{p_{v}\right\}$. Therefore, any iteration process that is stable will also be weakly stable but the converse is generally not true.

Definition 4.2 ([8]) Let $\left\{p_{v}\right\} \subset \mathcal{V}$ be a given sequence. Then, a sequence $\left\{h_{v}\right\} \subset \mathcal{V}$ is an approximate sequence of $\left\{p_{v}\right\}$ if, for any $c \in \mathbb{N}$, there exists $\eta=\eta(c)$ such that

$$
\left\|p_{v}-h_{v}\right\| \leq \eta, \quad \forall v \geq c
$$

Definition 4.3 ([8]) Let $\mathcal{U}: \mathcal{V} \rightarrow \mathcal{V}$ be a mapping and $\left\{p_{v}\right\}$ be an iterative procedure defined for $p_{1} \in \mathcal{V}$ and

$$
p_{v+1}=f\left(U, p_{v}\right), \quad v \geq 0 .
$$

Let $\left\{p_{v}\right\}$ converge to a fixed point $p^{\star}$ of $\mathcal{U}$. Suppose for any approximate sequence $\left\{h_{\nu}\right\} \subset \mathcal{V}$ of $\left\{p_{v}\right\}$

$$
\lim _{v \rightarrow \infty} \epsilon_{v}=\lim _{v \rightarrow \infty}\left\|h_{v+1}-f\left(u, h_{v}\right)\right\|=0 \Rightarrow \lim _{v \rightarrow \infty} h_{v}=p^{\star}
$$

then we shall say that (4.3) is weakly $\mathcal{U}$-stable or weakly stable with respect to $\mathcal{U}$.

In 2010, Timis [38] studied a new concept of weak stability that is obtained from Definition 4.3 by replacing the approximate sequence with the notion of the equivalent sequence that is more general. 
Definition 4.4 ([9]) Let $\left\{p_{v}\right\}$ and $\left\{h_{v}\right\}$ be two sequences. We say that these sequences are equivalent if

$$
\lim _{v \rightarrow \infty}\left\|p_{v}-h_{v}\right\|=0
$$

Definition 4.5 ([38]) Let $U: \mathcal{V} \rightarrow \mathcal{V}$ be a mapping. Let $\left\{p_{v}\right\}$ be an iterative procedure defined for $p_{1} \in \mathcal{V}$ and

$$
p_{v+1}=f\left(U, p_{v}\right), \quad v \geq 0 .
$$

Let $\left\{p_{v}\right\}$ converge to a fixed point $p^{\star}$ of $U$. Suppose for any equivalent sequence $\left\{h_{v}\right\} \subset \mathcal{V}$ of $\left\{p_{\nu}\right\}$

$$
\lim _{v \rightarrow \infty} \epsilon_{v}=\lim _{v \rightarrow \infty}\left\|h_{v+1}-f\left(u, h_{v}\right)\right\|=0 \Rightarrow \lim _{v \rightarrow \infty} h_{v}=p^{\star}
$$

then we shall say that (4.4) is weakly $w^{2}$-stable with respect to $u$.

It is shown in [38] with an example that any equivalent sequence is an approximative sequence but the reverse is not true.

In this section, we prove that the $\mathrm{AH}$ iterative scheme (1.6) is $w^{2}$-stable with respect to $u$ for contractive-like mappings.

Theorem 4.6 Suppose all the conditions in Theorem 3.1 hold. Then, the AH iterative scheme (1.6) is $w^{2}$-stable with respect to $u$.

Proof Let $\left\{h_{v}\right\} \subset \mathcal{V}$ be an equivalent sequence of $\left\{p_{v}\right\}$. Put $\epsilon_{v}=\left\|h_{v+1}-\left(1-k_{v}\right) a_{v}-k_{v} U a_{v}\right\|$, where $a_{v}=U\left(U b_{v}\right), b_{v}=U\left(U c_{v}\right), c_{v}=\left(1-r_{v}\right) h_{v}+r_{v} U h_{v}$. Suppose that $\lim _{v \rightarrow \infty} \epsilon_{v}=0$. Then, applying the triangular inequality, (1.2) and (1.6) give

$$
\begin{aligned}
& \left\|h_{v+1}-p^{\star}\right\| \leq\left\|h_{v+1}-p_{v+1}\right\|+\left\|p_{v+1}-p^{\star}\right\| \\
& \leq\left\|h_{v+1}-\left(1-k_{v}\right) a_{v}-k_{v} U a_{v}\right\| \\
& +\left\|\left(1-k_{v}\right) a_{v}+k_{v} u a_{v}-p_{v+1}\right\|+\left\|p_{v+1}-p^{\star}\right\| \\
& =\epsilon_{v}+\left\|\left(1-k_{v}\right) a_{v}-k_{v} u a_{v}-\left(1-k_{v}\right) q_{v}-k_{\nu} u q_{v}\right\| \\
& +\left\|p_{v+1}-p^{\star}\right\| \\
& \leq \epsilon_{v}+\left(1-k_{v}\right)\left\|q_{v}-a_{v}\right\|+k_{v}\left\|u q_{v}-U a_{v}\right\|+\left\|p_{v+1}-p^{\star}\right\| \\
& \leq \epsilon_{v}+\left(1-k_{v}\right)\left\|q_{v}-a_{v}\right\|+k_{v}\left[\zeta\left\|q_{v}-a_{v}\right\|+\psi\left(\| q_{v}-U q_{v}\right)\right] \\
& +\left\|p_{\nu+1}-p^{\star}\right\| \\
& \leq \epsilon_{v}+\left(1-(1-\zeta) k_{v}\right)\left\|q_{v}-a_{v}\right\| \\
& +k_{\nu} \psi\left(\left\|q_{v}-p^{\star}\right\|+\left\|u p^{\star}-u q_{v}\right\|\right)+\left\|p_{v+1}-p^{\star}\right\| \\
& \leq \epsilon_{v}+\left(1-(1-\zeta) k_{v}\right)\left\|q_{v}-a_{v}\right\| \\
& +k_{\nu} \psi\left((1+\zeta)\left\|q_{v}-p^{\star}\right\|\right)+\left\|p_{v+1}-p^{\star}\right\| .
\end{aligned}
$$


Since $\left(1-(1-\zeta) k_{v}\right)<1$ for all $v \in \mathbb{N}$, then from (4.5), we obtain

$$
\left\|h_{v+1}-p^{\star}\right\| \leq \epsilon_{v}+\left\|q_{v}-a_{v}\right\|+k_{v} \psi\left((1+\zeta)\left\|q_{v}-p^{\star}\right\|\right)+\left\|p_{v+1}-p^{\star}\right\| .
$$

Also,

$$
\begin{aligned}
\left\|q_{v}-a_{v}\right\|= & \left\|u\left(u_{w_{v}}\right)-U\left(U b_{v}\right)\right\| \\
\leq & \zeta\left\|u_{w_{v}}-u b_{v}\right\|+\psi\left(\left\|u_{w_{v}}-u\left(U w_{v}\right)\right\|\right) \\
\leq & \zeta\left(\zeta\left\|w_{v}-b_{v}\right\|+\psi\left(\left\|w_{v}-u_{w_{v}}\right\|\right)\right) \\
& +\psi\left(\zeta\left\|w_{v}-p^{\star}\right\|+\zeta\left\|u_{w_{v}}-p^{\star}\right\|\right) \\
\leq & \zeta^{2}\left\|w_{v}-b_{v}\right\|+\zeta \psi\left((1+\zeta)\left\|w_{v}-p^{\star}\right\|\right) \\
& +\psi\left(\zeta\left\|w_{v}-p^{\star}\right\|+\zeta^{2}\left\|w_{v}-p^{\star}\right\|\right) \\
= & \zeta^{2}\left\|w_{v}-b_{v}\right\|+\zeta \psi\left((1+\zeta)\left\|w_{v}-p^{\star}\right\|\right) \\
& +\psi\left(\zeta(1+\zeta)\left\|w_{v}-p^{\star}\right\|\right) .
\end{aligned}
$$

Similarly,

$$
\left\|w_{v}-b_{v}\right\| \leq \zeta^{2}\left\|z_{v}-c_{v}\right\|+\zeta \psi\left((1+\zeta)\left\|z_{v}-p^{\star}\right\|\right)+\psi\left(\zeta(1+\zeta)\left\|z_{v}-p^{\star}\right\|\right)
$$

Finally, since $\left(1-(1-\zeta) r_{v}\right)<1$ for all $v \in \mathbb{N}$, we have

$$
\begin{aligned}
\left\|z_{v}-c_{v}\right\| & \leq\left(1-r_{v}\right)\left\|p_{v}-h_{v}\right\|+r_{v}\left\|U p_{v}-U h_{v}\right\| \\
& \leq\left(1-r_{v}\right)\left\|p_{v}-h_{v}\right\|+r_{v} \zeta\left\|p_{v}-h_{v}\right\|+r_{v} \psi\left(\left\|p_{v}-U p_{v}\right\|\right) \\
& \leq\left(1-(1-\zeta) r_{v}\right)\left\|p_{v}-h_{v}\right\|+r_{v}(1+\zeta)\left\|p_{v}-p^{\star}\right\| \\
& \leq\left\|p_{v}-h_{v}\right\|+r_{v} \psi\left((1+\zeta)\left\|p_{v}-p^{\star}\right\|\right) .
\end{aligned}
$$

Using (4.6), (4.7), (4.8) and (4.9), we obtain

$$
\begin{aligned}
\left\|h_{v+1}-p^{\star}\right\| \leq & \epsilon_{v}+\zeta^{4}\left\|p_{v}-h_{v}\right\|+\zeta^{4} r_{v} \psi\left((1+\zeta)\left\|p_{v}-p^{\star}\right\|\right) \\
& +\zeta^{2} \psi\left((1+\zeta)\left\|z_{v}-p^{\star}\right\|\right)+\zeta^{2} \psi\left(\zeta(1+\zeta)\left\|w_{v}-p^{\star}\right\|\right) \\
& \left.+\psi\left((1+\zeta) \| w_{v}-p^{\star}\right)\right)+\psi\left(\zeta(1+\zeta)\left\|w_{v}-p^{\star}\right\|\right) \\
& +k_{v} \psi\left((1+\zeta)\left\|q_{v}-p^{\star}\right\|\right)+\left\|p_{v+1}-p^{\star}\right\| .
\end{aligned}
$$

We established in Theorem 3.1 that $\lim _{v \rightarrow \infty}\left\|p_{v}-p^{\star}\right\|=0$ and since $\psi$ is a strictly increasing continuous function with $\psi(0)=0$, consequently $\lim _{v \rightarrow \infty}\left\|p_{v+1}-p^{\star}\right\|=0$. Following the equivalence of $\left\{p_{v}\right\}$ and $\left\{h_{v}\right\}$, we have that $\lim _{v \rightarrow \infty}\left\|p_{v}-h_{v}\right\|=0$. Since $\lim _{v \rightarrow \infty} \epsilon_{v}=0$, then taking the limits of both sides of (4.10) yields $\lim _{v \rightarrow \infty}\left\|h_{v}-p^{\star}\right\|=0$. Hence, the AH iterative scheme (1.6) is $w^{2}$-stable with respect to $U$.

In order to support the analytical proof of Theorem 4.6, we provide the following illustrative example. 
Example 4.7 Let $(\mathbb{R},\|\cdot\|)$ be a Banach space with the usual norm and $\mathcal{V}=[0,1]$. Let $U$ : $\mathcal{V} \rightarrow \mathcal{V}$ be a mapping defined by $u p=\frac{p}{6}$. Then clearly, zero is the only fixed point of $U$ and $U$ satisfies (1.1) with $\zeta=\frac{1}{6}$.

Next, we show that the sequence $\left\{p_{v}\right\}$ generated by the iterative scheme (1.6) converges to $p^{\star}=0 \in \Im(\mathcal{J})$. For this, let $r_{v}=k_{v}=\frac{1}{v+3}$ and $p_{1} \in[0,1]$, then from (1.6), we obtain

$$
\begin{aligned}
z_{v} & =\left(1-\frac{1}{v+3}+\frac{1}{6(v+3)}\right) p_{v}=\left(1-\frac{5}{6(v+3)}\right) p_{v} \\
w_{v} & =\frac{1}{36}\left(1-\frac{5}{6(v+3)}\right) p_{v}, \\
q_{v} & =\frac{1}{1296}\left(1-\frac{5}{6(v+3)}\right) p_{v}, \\
p_{v+1} & =\frac{1}{1296}\left(1-\frac{10}{6(v+3)}+\frac{25}{36(v+3)^{2}}\right) p_{v} \\
& =\left[1-\left(\frac{1295}{1296}+\frac{10}{6^{5}(v+3)}+\frac{25}{6^{7}(v+3)^{2}}\right)\right] p_{v} .
\end{aligned}
$$

Set $y_{v}=\frac{1295}{1296}+\frac{10}{6^{5}(v+3)}+\frac{25}{6^{7}(v+3)^{2}}$. Note that $y_{v} \in(0,1)$ for all $v \in \mathbb{N}$ and $\sum_{v=0}^{\infty} y_{v}=\infty$. Thus, by Lemma 2.5, we obtain $\lim _{v \rightarrow \infty} p_{v}=0$.

It is not difficult to see that $\lim _{v \rightarrow \infty}\left\|p_{v}\right\|=\left\|\lim _{v \rightarrow \infty} p_{v}\right\|=0$. Then, if we take $h_{v}=\frac{1}{v+4}$ for all $v \in \mathbb{N}$, we obtain

$$
0 \leq \lim _{v \rightarrow \infty}\left\|p_{v}-h_{v}\right\| \leq \lim _{v \rightarrow \infty}\left\|p_{v}\right\|+\lim _{v \rightarrow \infty}\left\|h_{v}\right\|=0
$$

which shows that $\lim _{v \rightarrow \infty}\left\|p_{v}-h_{v}\right\|=0$. If follows that $\left\{p_{v}\right\}$ and $\left\{h_{v}\right\}$ are equivalent sequences.

Suppose that $\epsilon_{v}$ is the sequence associated with the iterative sequence $\left\{p_{v}\right\}$, then we obtain

$$
\begin{aligned}
\epsilon_{v} & =\left|h_{v+1}-\left(\frac{1}{1296}-\frac{10}{6^{5}(v+3)}+\frac{25}{6^{7}(v+3)^{2}}\right) h_{v}\right| \\
& =\left|\frac{1}{v+5}-\frac{1}{1296(v+4)}+\frac{10}{6^{5}(v+3)(v+4)}-\frac{25}{6^{7}(v+3)^{2}(v+4)}\right| .
\end{aligned}
$$

Clearly, $\lim _{v \rightarrow \infty} \epsilon_{v}=0$. Therefore, the sequence $\left\{p_{v}\right\}$ generated by the $\mathrm{AH}$ iterative scheme (1.6) is $w^{2}$-stable with respect $\mathcal{U}$.

\section{Convergence results}

In this section, we prove weak and strong convergence theorems of the $\mathrm{AH}$ iterative scheme (1.6) for Reich-Suzuki-type nonexpansive mappings.

Lemma 5.1 Let $U$ be a self Reich-Suzuki-type nonexpansive mapping defined on a nonempty closed convex subset $\mathcal{V}$ of a Banach space $\mathcal{M}$ with $\Im(U) \neq \emptyset$. Let $\left\{p_{v}\right\}$ be the sequence generated by the AH iterative scheme (1.6), then $\lim _{v \rightarrow \infty}\left\|p_{v}-p^{\star}\right\|$ exists for each $p^{\star} \in \Im(U)$. 
Proof Let $p^{\star} \in \Im(\mathcal{I})$. By Lemma 2.7, we obtain

$$
\begin{aligned}
\left\|z_{v}-p^{\star}\right\| & =\left\|\left(1-r_{v}\right) p_{v}+r_{v} U p_{v}-U p^{\star}\right\| \\
& \leq\left(1-r_{v}\right)\left\|p_{v}-p^{\star}\right\|+r_{v}\left\|U p_{v}-U p^{\star}\right\| \\
& \leq\left(1-r_{v}\right)\left\|p_{v}-p^{\star}\right\|+r_{v}\left\|p_{v}-p^{\star}\right\| \\
& =\left\|p_{v}-p^{\star}\right\|, \\
\left\|w_{v}-p^{\star}\right\| & =\left\|U\left(U z_{v}\right)-p^{\star}\right\| \\
& \leq\left\|U z_{v}-p^{\star}\right\| \\
& \leq\left\|z_{v}-p^{\star}\right\| \\
& \leq\left\|p_{v}-p^{\star}\right\|, \\
\left\|q_{v}-p^{\star}\right\| & =\left\|U\left(U w_{v}\right)-p^{\star}\right\| \\
& \leq\left\|w_{v}-p^{\star}\right\| \\
& \leq\left\|w_{v}-p^{\star}\right\| \\
& \leq\left\|p_{v}-p^{\star}\right\|, \\
\left\|p_{v+1}-p^{\star}\right\| & =\left\|\left(1-k_{v}\right) q_{v}+k_{v} U q_{v}-U p^{\star}\right\| \\
& \leq\left(1-k_{v}\right)\left\|q_{v}-p^{\star}\right\|+k_{v}\left\|U q_{v}-U p^{\star}\right\| \\
& \leq\left\|q_{v}-p^{\star}\right\| \\
& \leq\left\|p_{v}-p^{\star}\right\| .
\end{aligned}
$$

Thus, $\left\{\left\|p_{v}-p^{\star}\right\|\right\}$ is a bounded and decreasing sequence of reals and hence $\lim _{v \rightarrow \infty} \| p_{v}-$ $p^{\star} \|$ exists.

Lemma 5.2 Let $u$ be a self Reich-Suzuki-type nonexpansive mapping defined on a nonempty closed convex subset $\mathcal{V}$ of a uniformly convex Banach space $\mathcal{M}$. Let $\left\{p_{v}\right\}$ be the iterative sequence defined by the AH iterative scheme (1.6). Then, $\Im(U) \neq \emptyset$ if and only if $\left\{p_{v}\right\}$ is bounded and $\lim _{v \rightarrow \infty}\left\|u p_{v}-p_{v}\right\|=0$.

Proof Suppose that $\Im(U) \neq \emptyset$ and $p^{\star} \in \Im(U)$. Then, by Lemma $5.1, \lim _{v \rightarrow \infty}\left\|p_{v}-p^{\star}\right\|$ exists and $\left\{p_{v}\right\}$ is bounded. Now, we set

$$
\lim _{v \rightarrow \infty}\left\|p_{v}-p^{\star}\right\|=d .
$$

From (5.1), (5.2) and (5.5), we have

$$
\begin{aligned}
& \limsup _{v \rightarrow \infty}\left\|z_{v}-p^{\star}\right\| \leq d, \\
& \limsup _{v \rightarrow \infty}\left\|w_{v}-p^{\star}\right\| \leq d .
\end{aligned}
$$

Recalling Lemma 2.7, we have

$$
\underset{v \rightarrow \infty}{\limsup }\left\|u p_{v}-p^{\star}\right\| \leq \limsup _{v \rightarrow \infty}\left\|p_{v}-p^{\star}\right\|=d .
$$


Also, from (1.6) and Lemma 5.1, we obtain

$$
\begin{aligned}
\left\|p_{v+1}-p^{\star}\right\| & =\left\|\left(1-k_{v}\right) q_{v}+k_{v} U q_{v}-U p^{\star}\right\| \\
& \leq\left(1-k_{v}\right)\left\|q_{v}-p^{\star}\right\|+k_{v}\left\|U q_{v}-U p^{\star}\right\| \\
& \leq\left(1-k_{v}\right)\left\|p_{v}-p^{\star}\right\|+k_{v}\left\|q_{v}-p^{\star}\right\| \\
& \leq\left(1-k_{v}\right)\left\|p_{v}-p^{\star}\right\|+k_{v}\left\|U\left(U_{w_{v}}\right)-p^{\star}\right\| \\
& \leq\left(1-k_{v}\right)\left\|p_{v}-p^{\star}\right\|+k_{v}\left\|u_{w_{v}}-p^{\star}\right\| \\
& =\left(1-k_{v}\right)\left\|p_{v}-p^{\star}\right\|+k_{v}\left\|w_{v}-p^{\star}\right\| \\
& \leq\left(1-k_{v}\right)\left\|p_{v}-p^{\star}\right\|+k_{v}\left\|U\left(U z_{v}\right)-p^{\star}\right\| \\
& \leq\left(1-k_{v}\right)\left\|p_{v}-p^{\star}\right\|+k_{v}\left\|U z_{v}-p^{\star}\right\| \\
& \leq\left(1-k_{v}\right)\left\|p_{v}-p^{\star}\right\|+k_{v}\left\|z_{v}-p^{\star}\right\| \\
& =\left\|p_{v}-p^{\star}\right\|-k_{v}\left\|p_{v}-p^{\star}\right\|+k_{v}\left\|z_{v}-p^{\star}\right\| .
\end{aligned}
$$

This implies that

$$
\left\|p_{v+1}-p^{\star}\right\|-\left\|p_{v}-p^{\star}\right\| \leq \frac{\left\|p_{v+1}-p^{\star}\right\|-\left\|p_{v}-p^{\star}\right\|}{k_{v}} \leq\left\|z_{v}-p^{\star}\right\|-\left\|p_{v}-p^{\star}\right\| .
$$

Therefore,

$$
d \leq \liminf _{v \rightarrow \infty}\left\|z_{v}-p^{\star}\right\|
$$

From (5.6) and (5.9), we obtain

$$
d=\lim _{v \rightarrow \infty}\left\|z_{v}-p^{\star}\right\|
$$

Using (1.6), we have

$$
d=\lim _{v \rightarrow \infty}\left\|z_{v}-p^{\star}\right\|=\lim _{v \rightarrow \infty}\left\|\left(1-r_{v}\right)\left(p_{v}-p^{\star}\right)+r_{v}\left(u_{p_{v}}-p^{\star}\right)\right\| .
$$

Since $0<r_{v}<1$ for all $v \in \mathbb{N}$, then from Lemma 2.6, we have

$$
\lim _{v \rightarrow \infty}\left\|U p_{v}-p_{v}\right\|=0
$$

Conversely, suppose that $\left\{p_{v}\right\}$ is bounded and $\lim _{v \rightarrow \infty}\left\|\mathcal{U} p_{v}-p_{v}\right\|=0$. Let $p^{\star} \in A\left(\mathcal{V},\left\{p_{v}\right\}\right)$. By Lemma 2.8, we have

$$
\begin{aligned}
& r\left(U p^{\star},\left\{p_{v}\right\}\right)=\limsup _{v \rightarrow \infty}\left\|p_{v}-U p^{\star}\right\| \\
& \leq\left(\frac{3+\varpi}{1-\varpi}\right) \limsup _{v \rightarrow \infty}\left\|u p_{v}-p_{v}\right\|+\limsup _{v \rightarrow \infty}\left\|p_{v}-p^{\star}\right\| \\
& =\limsup _{v \rightarrow \infty}\left\|p_{v}-p^{\star}\right\| \\
& =r\left(p^{\star},\left\{p_{\nu}\right\}\right) \text {. }
\end{aligned}
$$


This implies that $U p^{\star} \in A\left(\mathcal{V},\left\{p_{v}\right\}\right)$. Since $\mathcal{M}$ is uniformly convex, then $A\left(\mathcal{V},\left\{p_{v}\right\}\right)$ has only one element, therefore we obtain $U p^{\star}=p^{\star}$.

Now, we establish the weak convergence result. For this, the following Lemma will be useful:

Lemma 5.3 If all the assumptions in Theorem 5.4 are fulfilled, then $\lim _{v \rightarrow \infty}\left\langle p_{v}, J\left(p_{1}^{\star}-p_{2}^{\star}\right)\right\rangle$ exists for any $p_{1}^{\star}, p_{2}^{\star} \in \Im(U)$; in particular $\lim _{v \rightarrow \infty}\left\langle p-q, J\left(p_{1}^{\star}-p_{2}^{\star}\right)\right\rangle=0$ for all $p, q \in \omega_{w}\left(p_{v}\right)$, where $\omega_{w}\left(p_{v}\right)$ denotes the set of all weak limit points of $\left\{p_{v}\right\}$.

Proof The conclusion follows from Lemma 2.3 in [20].

Theorem 5.4 Let $\mathcal{U}, \mathcal{V}$ and $p_{v}$ be as in Lemma 5.2. Let $\mathcal{M}$ be a uniformly convex Banach space. Suppose that either of the following assumptions holds:

(a) $\mathcal{M}$ satisfies Opial's condition and I $-\mathcal{U}$ is demiclosed with respect to zero;

(b) $\mathcal{M}$ has a Fréchet differential norm.

If $\Im(U) \neq \emptyset$, then the sequence $\left\{p_{v}\right\}$ converges weakly to a point of $U$.

Proof By Lemma 5.1, we have that $\lim _{v \rightarrow \infty}\left\|p_{v}-p^{\star}\right\|$ exists. Now, it is sufficient to prove that $\left\{p_{v}\right\}$ have a unique weak subsequential limit in $\Im(U)$. Suppose that $\left\{p_{v_{i}}\right\}$ and $\left\{p_{v_{k}}\right\}$ are two subsequences of $\left\{p_{v}\right\}$, which converge weakly to $g$ and $y$, respectively. Now, suppose that (a) is true. Then, from Lemma 5.2, $\lim _{v \rightarrow \infty}\left\|U p_{v}-p_{v}\right\|=0$ and by the demiclosedness of $I-U$ with respect to zero, we have that $(1-U) g=0$. That is, $g=U g$; similarly $y=U y$.

Next, we prove uniqueness. Since $g, y \in \Im(U)$, then $\lim _{v \rightarrow \infty}\left\|p_{v}-g\right\|$ and $\lim _{v \rightarrow \infty}\left\|p_{v}-y\right\|$ exists. If $g \neq y$, then from Opial's condition, we have

$$
\begin{aligned}
\lim _{v \rightarrow \infty}\left\|p_{v}-g\right\| & =\lim _{v_{i} \rightarrow \infty}\left\|p_{v_{i}}-g\right\|<\lim _{v_{i} \rightarrow \infty}\left\|p_{v_{i}}-y\right\|=\lim _{v \rightarrow \infty}\left\|p_{v}-y\right\| \\
& =\lim _{v_{k} \rightarrow \infty}\left\|p_{v_{k}}-y\right\|<\lim _{v_{k} \rightarrow \infty}\left\|p_{v_{k}}-g\right\|=\lim _{v \rightarrow \infty}\left\|p_{v}-g\right\|,
\end{aligned}
$$

which is a contradiction, so $g=y$. Again, assume that (b) holds. Recalling Lemma 5.3, we have $\left\langle p_{v}, J\left(p_{1}^{\star}-p_{2}^{\star}\right)\right\rangle=0$ for all $p, q \in \omega_{w}\left(p_{v}\right)$. Thus, $\|g-y\|^{2}=\langle g-y, J(g-y)\rangle$ implies $g=y$.

We now establish the following strong convergence results:

Theorem 5.5 Let $\mathcal{U}, \mathcal{V}$ and $\mathcal{M}$ be as in Lemma 5.2. The sequence $\left\{p_{v}\right\}$ generated by the $A H$ iterative scheme (1.6) converges to an element of $\Im(U)$ if and only if $\liminf _{v \rightarrow \infty} d\left(p_{v}, \Im(U)\right)=$ 0 , where $d\left(p_{v}, \Im(\mathcal{I})\right)=\inf \left\{\left\|p_{v}-p^{\star}\right\|: p^{\star} \in \Im(U)\right\}$.

Proof The necessity is obvious.

Conversely, suppose $\liminf _{v \rightarrow \infty} d\left(p_{v}, \Im(U)\right)=0$ and $p^{\star} \in \mathfrak{\Im}(\mathcal{U})$. By Lemma 5.1, $\lim _{v \rightarrow \infty}\left\|p_{v}-p^{\star}\right\|$ exists, for any $p^{\star} \in \Im(U)$. It is sufficient to prove that the sequence $\left\{p_{v}\right\}$ is Cauchy in $\mathcal{V}$. Since $\lim _{v \rightarrow \infty} d\left(p_{v}, \Im(\mathcal{I})\right)=0$, then given $\varepsilon>0$, there exists $\rho_{0} \in \mathbb{N}$ such that for all $v \geq \rho_{0}$

$$
\begin{aligned}
& d\left(p_{v}, \Im(U)\right)<\frac{\varepsilon}{2}, \\
& \inf \left\{\left\|p_{v}-p^{\star}\right\|: p^{\star} \in \Im(U)\right\}<\frac{\varepsilon}{2} .
\end{aligned}
$$


In particular, $\inf \left\{\left\|p_{\rho_{0}}-p^{\star}\right\|: p^{\star} \in \Im(U)\right\}<\frac{\varepsilon}{2}$. Therefore, there exists $p^{\star} \in \Im(U)$ such that

$$
\left\|p_{\rho_{0}}-p^{\star}\right\|<\frac{\varepsilon}{2} .
$$

Now for $\rho, v \geq \rho_{0}$, we have

$$
\begin{aligned}
\left\|p_{v+\rho}-p_{v}\right\| & \leq\left\|p_{v+\rho}-p^{\star}\right\|+\left\|p_{v}-p^{\star}\right\| \\
& \leq\left\|p_{\rho_{0}}-p^{\star}\right\|+\left\|p_{\rho_{0}}-p^{\star}\right\| \\
& =2\left\|p_{\rho_{0}}-p^{\star}\right\|<\varepsilon .
\end{aligned}
$$

This implies that the sequence $\left\{p_{v}\right\}$ is Cauchy in $\mathcal{V}$. Since $\mathcal{V}$ is closed, there must be an element $q \in \mathcal{V}$ such that $\lim _{v \rightarrow \infty} p_{v}=q$. Now, $\lim _{v \rightarrow \infty} d\left(p_{v}, \mathfrak{s}(\mathcal{U})\right)=0$ gives that $d(q, \Im)(U)=$ 0 , that is $q \in \Im(U)$.

A strong convergence on a compact domain is established in the following way:

Theorem 5.6 Let $\mathcal{U}$ and $\mathcal{M}$ be as in Lemma 5.2 and $\mathcal{V}$ be a nonempty compact convex subset of $\mathcal{M}$. Then, the sequence $\left\{p_{v}\right\}$ generated by the iterative scheme (1.6) converges strongly to a fixed point of $U$.

Proof According to Lemma 5.2, $\lim _{v \rightarrow \infty}\left\|u_{p_{v}}-p_{v}\right\|=0$. Since $\mathcal{V}$ is convex and compact, the iterative sequence $\left\{p_{v}\right\}$ contained in the set $\mathcal{V}$ has a convergent subsequence, namely, $\left\{p_{v_{i}}\right\}$ endowed with a strong limit, namely, $q \in \mathcal{V}$. Putting $p=p_{v_{i}}$ and $q=q$, we apply Lemma 2.8 , to obtain

$$
\left\|p_{v_{i}}-u q\right\| \leq\left(\frac{3+\varpi}{1-\varpi}\right)\left\|p_{v_{i}}-u p_{v_{i}}\right\|+\left\|p_{v_{i}}-q\right\| .
$$

As $i \rightarrow \infty$, one can see that $p_{v_{i}} \rightarrow U q$. It follows that $U q=q$, i.e., $q \in \mathfrak{I}(\mathcal{U})$. According to Lemma 5.1, $\lim _{v \rightarrow \infty}\left\|p_{v}-q\right\|$ exists, that is, $q$ forms a strong limit for $\left\{p_{v}\right\}$.

A strong convergence theorem using a condition (I) of the operators is the following:

Theorem 5.7 Let $U, \mathcal{V}$ and $\mathcal{M}$ be as in Lemma 5.2. If $U$ satisfies condition (I), then the sequence $\left\{p_{v}\right\}$ generated by the AH iterative scheme (1.6) converges strongly to a fixed point

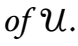

Proof In Lemma 5.2, we have shown that

$$
\lim _{v \rightarrow \infty}\left\|U p_{v}-p_{v}\right\|=0 .
$$

By Definition 2.4 and (5.11), we have

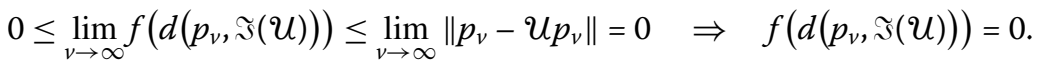

Since $f:[0, \infty) \rightarrow[0, \infty)$ is a nondecreasing function that satisfies the condition $f(0)=0$ and $f(s)>0$, for all $s>0$, we obtain

$$
\lim _{v \rightarrow \infty} d\left(p_{v}, \Im(\mathcal{\Im})\right)=0
$$


Since all the requirements of Theorem 5.5 are shown, one concludes that the sequence $\left\{p_{v}\right\}$ is strongly convergent in the fixed-point set of $\mathcal{U}$.

\section{Numerical result}

In this section, we give an example of a Reich-Suzuki-type nonexpansive mapping that does not satisfy the condition $(\mathrm{C})$. Further, we compare the convergence of the AH iterative scheme with some leading iterative schemes in the literature.

Example 6.1 Let $(\mathbb{R},\|\cdot\|)$ be a Banach space with the usual norm and $\mathcal{V}=[5,7]$. Let $\mathcal{u}$ : $\mathcal{V} \rightarrow \mathcal{V}$ be a mapping defined by

$$
U p= \begin{cases}\frac{p+20}{5}, & \text { if } p<7 \\ 4, & \text { if } p=7\end{cases}
$$

(i) The mapping $U$ does not satisfy the condition (C). For this, let $p=6$ and $q=7$, we have

$$
\frac{1}{2}|p-U p|=\frac{1}{2}|6-U(6)|=\frac{2}{5}<1=|p-q| .
$$

On the other hand,

$$
|u p-U q|=|u(6)-U(7)|=\frac{6}{5}>1=|p-q| .
$$

(ii) Now, to demonstrate that $U$ is a Reich-Suzuki-type nonexpasive mapping, the following cases are considered.

Case 1: If $p, q<7$, then

$$
\begin{aligned}
& \varpi|p-U p|+\varpi|q-U q|+(1-2 \varpi)|p-q| \\
& =\frac{1}{2}\left|p-\left(\frac{p+20}{5}\right)\right|+\frac{1}{2}\left|q-\left(\frac{p+20}{5}\right)\right| \\
& =\frac{1}{2}\left|\frac{4 p-20}{6}\right|+\frac{1}{2}\left|\frac{4 q-20}{5}\right| \\
& \geq \frac{1}{2}\left|\left(\frac{4 p-20}{5}\right)-\left(\frac{4 q-20}{5}\right)\right| \\
& =\frac{1}{2}\left|\frac{4 p}{5}-\frac{4 p}{5}\right|=\frac{2}{5}|p-q| \\
& \geq \frac{1}{5}|p-q|=\mid u_{p}-u_{q} .
\end{aligned}
$$

Case 2: If $p<7$ and $q=7$, then we have

$$
\begin{aligned}
\varpi\left|p-U_{p}\right|+\varpi\left|q-u_{q}\right|+(1-2 \varpi)|p-q| & =\frac{1}{2}\left|\frac{4 p-20}{5}\right|+\frac{1}{2}|7-4| \\
& =\frac{1}{2}\left|\frac{4 p-20}{5}\right|+\frac{3}{2} \\
& \geq\left|\frac{p}{5}\right|=\left|u_{p}-u_{q}\right| .
\end{aligned}
$$


Case 3: If $q<7$ and $p=7$, then we obtain

$$
\begin{aligned}
\varpi|p-U p|+\varpi|q-U q|+(1-2 \varpi)|p-q| & =\frac{1}{2}|7-4|+\frac{1}{2}\left|\frac{4 q-20}{5}\right| \\
& =\frac{3}{2}+\frac{1}{2}\left|\frac{4 q-20}{5}\right| \\
& \geq\left|\frac{q}{5}\right|=\left|u_{p}-u_{q}\right| .
\end{aligned}
$$

Case 4: If $p=q=7$, then we obtain

$$
\varpi\left|p-u_{p}\right|+\varpi\left|q-u_{q}\right|+(1-2 \varpi)|p-q| \geq 0=|4-4|=\left|u_{p}-u_{q}\right| .
$$

Hence, $\mathcal{U}$ is a Reich-Suzuki-type nonexpansive mapping and has fixed point 5 .

From Table 4 and Fig. 2, it can be clearly seen that the AH iterative converges faster to the fixed point of $U$ than other iterative schemes.

Table 4 Comparison of convergence behavior of AH (1.6) with S, Abbas, Thakur and JK iterative schemes

\begin{tabular}{rlllll}
\hline Step & S & Abbas & Thakur & JK & AH \\
\hline 1 & 5.5000000 & 5.5000000 & 5.5000000 & 5.5000000 & 5.5000000 \\
2 & 5.0993388 & 5.0602104 & 5.0198678 & 5.0171967 & 5.0006879 \\
3 & 5.0197364 & 5.0072506 & 5.0007895 & 5.0005915 & 5.00000009 \\
4 & 5.0039212 & 5.0008731 & 5.0000314 & 5.0000203 & 5.0000000 \\
5 & 5.0007791 & 5.0001051 & 5.0000012 & 5.0000007 & 5.0000000 \\
6 & 5.0001548 & 5.0000127 & 5.0000000 & 5.0000000 & 5.0000000 \\
7 & 5.0000308 & 5.0000015 & 5.0000000 & 5.0000000 & 5.0000000 \\
8 & 5.0000061 & 5.0000002 & 5.0000000 & 5.0000000 & 5.0000000 \\
9 & 5.0000012 & 5.0000000 & 5.0000000 & 5.0000000 & 5.0000000 \\
10 & 5.0000002 & 5.0000000 & 5.0000000 & 5.0000000 & 5.0000000 \\
11 & 5.0000000 & 5.0000000 & 5.0000000 & 5.0000000 & 5.00000000 \\
\hline
\end{tabular}

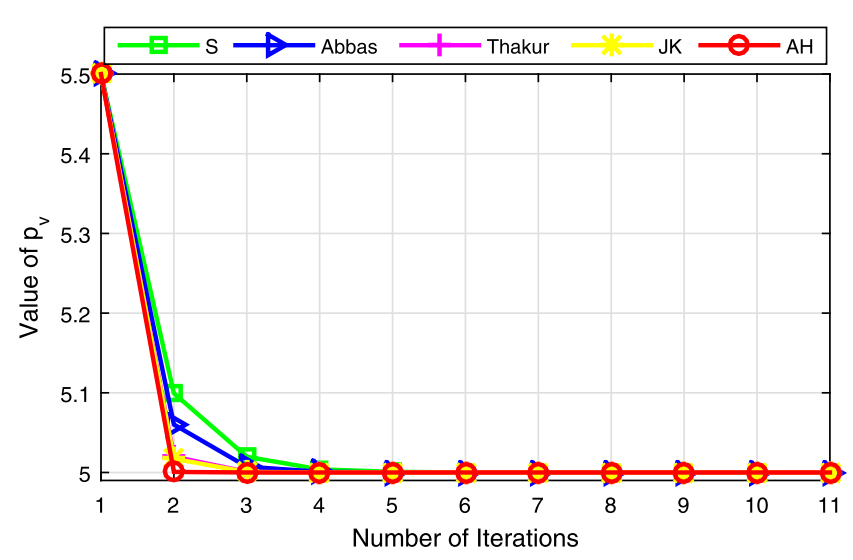

Figure 2 Graph corresponding to Table 4 


\section{Application}

In this section, we consider the application of our main results to the following nonlinear mixed Volterra-Fredholm-type integral equation:

$$
p(t)=\vartheta(t)+\lambda \int_{a}^{t} \int_{a}^{b} \varphi(r, s) \Upsilon(s, p(s)) d s d r, \quad t \in[a, b]
$$

Let $C([a, b])$ denote the space of all real-valued continuous functions on $I=[a, b]$. It is well known that $C([a, b])$ is a Banach space with the maximum norm,

$$
\|p-q\|_{\infty}=\max _{t \in[a, b]}|p(t)-q(t)|, \quad \forall p, q \in C([a, b]) .
$$

Theorem 7.1 Let $\mathcal{H}$ be a nonempty closed convex subset of $\mathcal{G}=C([a, b])$ and $\mathcal{U}: \mathcal{H} \rightarrow \mathcal{H}$ be defined by

$$
u_{p}(t)=\vartheta(t)+\lambda \int_{a}^{t} \int_{a}^{b} \varphi(r, s) \Upsilon(s, p(s)) d s d r, \quad t \in[a, b], \lambda \geq 0 .
$$

Assume that the following conditions hold:

$\left(Z_{1}\right) \vartheta: I \rightarrow \mathbb{R}$ is continuous;

$\left(Z_{2}\right) \varphi: I \times I \rightarrow \mathbb{R}$ is continuous for all $(r, s) \in I \times I$ such that $|\varphi(s, r)| \leq M$;

$\left(Z_{3}\right) \Upsilon: I \times \mathcal{H} \rightarrow \mathcal{H}$ is continuous and there exists a constant $L_{\Upsilon}>0$ such that

$$
\left|\Upsilon\left(s, p_{1}\right)-\Upsilon\left(s, p_{2}\right)\right| \leq L_{\Upsilon}\left|p_{1}-q_{2}\right|
$$

for all $s \in I$ and $p_{1}, q_{2} \in \mathbb{R}$

$\left(Z_{4}\right) \lambda L_{\Upsilon} M(b-a)^{2}<1$.

Then, the mixed Volterra-Fredholm integral equation (7.1) has a unique solution in $C([a, b])$ if and only if $U$ admits an a.f.p.s.

Proof Let $p, q \in C([a, b])$, then

$$
\begin{aligned}
\|p-U q\|_{\infty}= & \max _{t \in[a, b]}|p(t)-u q(t)| \\
= & \max _{t \in[a, b]} \mid p(t)-\vartheta(t)-\lambda \int_{a}^{t} \int_{a}^{b} \varphi(r, s) \Upsilon(s, p(s)) d s d r+\vartheta(t) \\
& +\lambda \int_{a}^{t} \int_{a}^{b} \varphi(r, s) \Upsilon(s, p(s)) d s d r-\vartheta(t) \lambda \int_{a}^{t} \int_{a}^{b} \varphi(r, s) \Upsilon(s, q(s)) d s d r \mid \\
\leq & \max _{t \in[a, b]}|p(t)-U p(t)| \\
& +\lambda \max _{t \in[a, b]}\left|\int_{a}^{t} \int_{a}^{b} \varphi(r, s) \Upsilon(s, p(s)) d s d r-\int_{a}^{t} \int_{a}^{b} \varphi(r, s) \Upsilon(s, q(s)) d s d r\right| \\
\leq & \max _{t \in[a, b]}\left|p(t)-U_{p}(t)\right|+\lambda L_{\Upsilon} \max _{t \in[a, b]} \int_{a}^{t} \int_{a}^{b}|\varphi(r, s)||p(s)-q(s)| d s d r \\
\leq & \left\|p-U_{p}\right\|_{\infty}+\lambda L_{\Upsilon}\|p-q\|_{\infty} \max _{t \in[a, b]} \int_{a}^{t} \int_{a}^{b}|\varphi(r, s)| d s d r \\
\leq & \left\|p-U_{p}\right\|_{\infty}+\lambda L_{\Upsilon} M(b-a)^{2}\|p-q\|_{\infty} .
\end{aligned}
$$


By assumption $\left(Z_{3}\right)$, we have $\lambda L_{\Upsilon} M(b-a)^{2}<1$, then (7.2) yields

$$
\left\|p-u_{q}\right\|_{\infty} \leq\left\|p-u_{p}\right\|_{\infty}+\|p-q\|_{\infty}
$$

Therefore, by Lemma 2.8, $\mathcal{U}$ is a Reich-Suzuki-type nonexpansive mapping since it satisfies the condition (2.4) on $\mathcal{H}$ with $\frac{3+\varpi}{1-\varpi}=1$.

Take $\mathcal{H}=\mathcal{V}$ and $\mathcal{G}=\mathcal{M}$. Then, all the conditions of Lemma 5.2 are fulfilled, thus, (7.1) has a solution in $\mathcal{H} \subseteq C([a, b])$.

Example 7.2 Consider the following mixed-type nonlinear integral equation:

$$
p(t)=\frac{\pi}{2} t-\frac{t^{2}}{7 \pi}+\frac{2}{7} \int_{0}^{t} \int_{0}^{1} r \frac{\sin (p(s))}{2} d t d r, \quad t \in[0,1] .
$$

Clearly, the above integral equation is a special case of (7.1) with

$$
\vartheta(t)=\frac{\pi}{2} t-\frac{t^{2}}{7 \pi}, \quad \varphi(r, s)=r \quad \text { and } \quad \Upsilon(t, p(s))=\frac{\sin (p(s))}{2}
$$

Then, for any $t \in[0,1]$ and $p_{1}, q_{1} \in \mathbb{R}$, we obtain

$$
\left|\Upsilon\left(t, p_{1}\right)-\Upsilon\left(t, q_{1}\right)\right|=\frac{1}{2}\left|\sin p_{1}-\sin q_{1}\right|
$$

Next, for any $p, q \in \mathbb{R}$ with $p<q$, then by the mean-value theorem, there exists $f, p<f<q$ such that $\frac{\cos p-\cos q}{p-q}=-\sin f \Rightarrow|\cos p-\cos q| \leq|p-q|$. Therefore, (7.4) yields

$$
\left|\Upsilon\left(t, p_{1}\right)-\Upsilon\left(t, q_{1}\right)\right| \leq \frac{1}{2}\left|p_{1}-q_{1}\right|
$$

It is not difficult to see that $\vartheta(t)$ is continuous on $[0,1]$. Also, $\Upsilon(t, p(s))$ is continuous and $(r, s) \in[0,1] \times[0,1]$, thus

$$
M=\max _{(r, s) \in[0,1] \times[0,1]}|r|=1 .
$$

Consequently, all the assumptions in Theorem 7.1 are performed with $\lambda L_{\Upsilon} M(b-a)^{2}=$ $\frac{2}{7} \cdot \frac{1}{2} \cdot 1 \cdot(1-0)^{2}=\frac{2}{14}<1$.

Hence, there exists a solution of the mixed-type nonlinear integral equation (7.3). Further, one can easily verify that the function $p(t)=\frac{\pi}{2} t$ is the exact solution of (7.3).

\section{Conclusion}

In this work, we have introduced a four-step iterative scheme, called the $\mathrm{AH}$ iterative scheme (1.6) for approximating the fixed points of contractive-like mappings and ReichSuzuki-type nonexpansive mappings. The new iterative scheme has been shown to converge faster than the JK iterative scheme (1.5) analytically for contractive-like mappings. Furthermore, we have illustrated numerically that our new iterative scheme converges faster than many prominent iterative schemes in the literature for contractive-like mappings. The $w^{2}$-stability result of the $\mathrm{AH}$ iterative scheme has also been established for 
contractive-like mappings. We have provided an example to illustrate the notion of $w^{2}$ stability of the AH iterative scheme with respect to $U$. Also, we have proved weak and several strong convergence theorems for Reich-Suzuki-type nonexpansive mappings in uniformly convex Banach spaces. A new example of Reich-Suzuki-type nonexpansive mappings has been provided to compare the convergence behavior of the $\mathrm{AH}$ iterative method (1.6) with some well-known iterative schemes. As an application, we used our main results to establish the existence of solution of a mixed-type nonlinear integral equation. Finally, we illustrated the result in our application with an interesting example.

\author{
Acknowledgements \\ The authors are thankful to the editor and anonymous referees for their valuable comments and suggestions. \\ Funding \\ This research received no external funding. \\ Availability of data and materials \\ The data used to support the findings of this study are available from the corresponding author upon request.
}

\title{
Declarations
}

\section{Competing interests}

The authors declare that they have no competing interests.

\section{Authors' contributions}

AEO analyzed the results in the literature and made the original draft preparation. HI carried out the formal analysis, writing review and editing, and project administration. FA performed the validation and formal analysis. JA conducted the formal analysis, writing review and editing. All the authors read and approved the final manuscript.

\section{Author details}

${ }^{1}$ Department of Mathematics, University of Uyo, Uyo, Nigeria. ${ }^{2}$ Department of Engineering Science, Bandırma Onyedi Eylül University, Bandırma 10200, Balıkesir, Turkey. ${ }^{3}$ Department of Mathematics, Maulana Azad National Institute of Technology, Bhopal 462003, India. ${ }^{4}$ Department of Mathematics and Statistics, International Islamic University, H-10, Islamabad 44000, Pakistan.

\section{Publisher's Note}

Springer Nature remains neutral with regard to jurisdictional claims in published maps and institutional affiliations.

Received: 20 October 2021 Accepted: 7 February 2022 Published online: 02 March 2022

\section{References}

1. Abbas, M., Nazir, T.: A new faster iteration process applied to constrained minimization and feasibility problems. Mat. Vesn. 66, 223-234 (2014)

2. Agarwal, R.P., O'Regan, D., Sahu, D.R.: Iterative construction of fixed points of nearly asymptotically nonexpansive mappings. J. Nonlinear Convex Anal. 8, 61-79 (2007)

3. Ahmad, J., Ullah, K., Arshad, M., Ma, Z.: A new iterative method for Suzuki mappings in Banach spaces. J. Math. 2021, Article ID 6622931 (2021). https://doi.org/10.1155/2021/6622931

4. Ali, F., Ali, J., Nieto, J.J.: Some observations on generalized non-expansive mappings with an application. Comput. Appl. Math. 39(2), 74 (2020)

5. Amnuaykarn, K., Kumam, P., Nantadilok, J.: On the existence of best proximity points of multi-valued mappings in CAT(0) spaces. J. Nonlinear Funct. Anal. 2021, Article ID 25 (2021)

6. Berinde, $\mathrm{V}$ : On the approximation of fixed points of weak contractive mappings. Carpath. J. Math. 19, 7-22 (2003)

7. Berinde, V.: Picard iteration converges faster than Mann iteration for a class of quasicontractive operators. Fixed Point Theory Appl. 2, 97-105 (2004)

8. Berinde, V.: Iterative Approximation of Fixed Points. Springer, Berlin (2007)

9. Cardinali, T., Rubbioni, P.: A generalization of the Caristi fixed point theorem in metric spaces. Fixed Point Theory 11, 3-10 (2010)

10. Ceng, L.C., Petrusel, A., Yao, J.C., Yao, Y.: Systems of variational inequalities with hierarchical variational inequality constraints for Lipschitzian pseudocontractions. Fixed Point Theory 20, 113-133 (2019)

11. Chugh, R., Kumar, V., Kumar, S.: Strong convergence of a new three step iterative scheme in Banach spaces. Am. J. Comput. Math. 2, 345-357 (2012)

12. Goebel, K., Kirk, W.A.: Topics in Metric Fixed Point Theory. Cambridge University Press, Cambridge (1990)

13. Gursoy, F., Karakaya, V.: A Picard-S hybrid type iteration method for solving a differential equation with retarded argument (2014) 1403.2546v2

14. Harder, A.M.: Fixed point theory and stability results for fixed-point iteration procedures. Ph.D. thesis, University of Missouri-Rolla, Missouri (1987) 
15. Harder, A.M., Hicks, T.L.: A stable iteration procedure for nonexpansive mappings. Math. Jpn. 33, 687-692 (1988)

16. Hassan, S., De la Sen, M., Agarwal, P., Ali, Q., Hussain, A.: A new faster iterative scheme for numerical fixed points estimation of Suzuki's generalized nonexpansive mappings. Math. Probl. Eng. 2020, Article ID 3863819 (2020). https://doi.org/10.1155/2020/3863819

17. Imoru, C.O., Olantiwo, M.O.: On the stability of Picard and Mann iteration processes. Carpath. J. Math. 19(2), 155-160 (2003)

18. Iqbal, H., Abbas, M., Husnine, S.M.: Existence and approximation of fixed points of multivalued generalized $\alpha$-nonexpansive mappings in Banach spaces. Numer. Algorithms (2020). https://doi.org/10.1007/s1 1075-019-00854-z

19. Ishikawa, S.: Fixed points by a new iteration method. Proc. Am. Math. Soc. 44, 147-150 (1974)

20. Khan, S.H., Kim, J.K.: Common fixed points of two nonexpansive mappings by a modified faster iteration scheme. Bull. Korean Math. Soc. 47(5), 973-985 (2010)

21. Mann, W.R.: Mean value methods in iteration. Proc. Am. Math. Soc. 4, 506-510 (1953)

22. Noor, M.A.: New approximation schemes for general variational inequalities. J. Math. Anal. Appl. 251, 217-229 (2000)

23. Ofem, A.E., Igbokwe, D.I: An efficient iterative method and its applications to a nonlinear integral equation and a delay differential equation in Banach spaces. Turk. J. Inequal. 4(2), 79-107 (2020)

24. Ofem, A.E., Igbokwe, D.I: New faster four step iterative algorithm for Suzuki generalized nonexpansive mappings with an application. Adv. Theory Nonlinear Anal. Appl. 5(4), 482-506 (2021)

25. Ofem, A.E., Udofia, U.E.: Iterative solutions for common fixed points of nonexpansive mappings and strongly pseudocontractive mappings with applications. Can. J. Appl. Math. 3(1), 18-36 (2021)

26. Ofem, A.E., Udofia, U.E., Igbokwe, D.I.: New iterative for solving constrained convex minimization problem and split feasibility problem. Eur. J. Math. Anal. 1(2), 106-132 (2021)

27. Okeke, G.A., Abbas, M., Dela Sen, M.: Approximation of the fixed point of multivalued quasi-nonexpansive mappings via a faster iterative process with applications. Discrete Dyn. Nat. Soc. 2020, Article ID 8634050 (2020). https://doi.org/10.1155/2020/8634050

28. Osilike, M.O., Udomene, A.: Short proofs of stability results for fixed-point iteration procedures for a class of contractive-type mappings. Indian J. Pure Appl. Math. 30(12), 1229-1234 (1999)

29. Pant, R., Pandey, R.: Existence and convergence results for a class of non-expansive type mappings in hyperbolic spaces. Appl. Gen. Topol. 20, 281-295 (2019)

30. Reich, S., Zaslavski, A.J.: Fixed points and convergence results for a class of contractive mappings. J. Nonlinear Var. Anal. 5, 665-671 (2021)

31. Reich, S., Zaslavski, A.J.: Convergence of iterates of nonlinear contractive mappings. Appl. Set-Valued Anal. Optim. 3, 109-115 (2021)

32. Sahu, D.R., Petrusel, A.: Strong convergence of iterative methods by strictly pseudocontractive mappings in Banach spaces. Nonlinear Anal. 74, 6012-6023 (2011)

33. Schu, J.: Weak and strong convergence to fixed points of asymptotically nonexpansive mappings. Bull. Aust. Math. Soc. 43, 153-159 (1991)

34. Senter, H.F., Dotson, W.G.: Approximating fixed points of nonexpansive mapping. Proc. Am. Math. Soc. 44, 375-380 (1974)

35. Suzuki, T.: Fixed point theorems and convergence theorems for some generalized nonexpansive mappings. J. Math. Anal. Appl. 340, 1088-1095 (2008)

36. Takahashi, W., Wen, C.F., Yao, J.C.: Strong convergence theorems by hybrid methods for noncommutative normally 2-generalized hybrid mappings in Hilbert spaces. Appl. Anal. Optim. 3, 43-56 (2019)

37. Thakur, B.S., Thakur, D., Postolache, M.: A new iterative scheme for numerical reckoning fixed points of Suzuki's generalized nonexpansive mappings. Appl. Math. Comput. 275, 147-155 (2016)

38. Timis, I.: On the weak stability of Picard iteration for some contractive type mappings and coincidence theorems. Int. J. Comput. Appl. 37, 9-13 (2012)

39. Ullah, K., Ahmad, J., Arshad, M., Ma, Z.: Approximating fixed points using a faster iterative method and application to split feasibility problems. Comput. 2021 (2021). https://doi.org/10.3390/computation9080090

40. Ullah, K., Ahmad, J., de la Sen, M.: On generalized nonexpansive maps in Banach spaces. Comput. 2020 (2020). https://doi.org/10.3390/computation8030061

41. Ullah, K., Arshad, M.: Numerical reckoning fixed points for Suzuki's generalized nonexpansive mappings via new iteration process. Filomat 32, 187-196 (2018)

42. Weng, X.: Fixed point iteration for local strictly pseudocontractive mapping. Proc. Am. Math. Soc. 113, 727-731 (1991)

43. Yao, Y., Liou, Y.C., Yao, J.C.: Split common fixed point problem for two quasi-pseudo-contractive operators and its algorithm construction. Fixed Point Theory Appl. 2015, 127 (2015)

44. Yao, Y., Liou, Y.C., Yao, J.C.: Iterative algorithms for the split variational inequality and fixed point problems under nonlinear transformations. J. Nonlinear Sci. Appl. 10, 843-854 (2017)

45. Yao, Y., Qin, X., Yao, J.C.: Projection methods for firmly type nonexpansive operators. J. Nonlinear Convex Anal. 19 407-415 (2018)

46. Yao, Y., Yao, J.C., Liou, Y.C., Postolache, M.: Iterative algorithms for split common fixed points of demicontractive operators without priori knowledge of operator norms. Carpath. J. Math. 34, 459-466 (2018)

47. Zamfirescu, T.: Fixed point theorems in metric spaces. Arch. Math. (Basel) 23, 292-298 (1972) 